ESAIM: M2AN

Vol. 42, $\mathrm{N}^{\mathrm{O}} 1,2008$, pp. 141-174

DOI: $10.1051 / \mathrm{m} 2 \mathrm{an}: 2007056$
ESAIM: Mathematical Modelling and Numerical Analysis

www.esaim-m2an.org

\title{
A FULL DISCRETIZATION OF THE TIME-DEPENDENT NAVIER-STOKES EQUATIONS BY A TWO-GRID SCHEME
}

\author{
Hyam ABboud $^{1,2}$ AND TONI SAYAH ${ }^{2}$
}

\begin{abstract}
We study a two-grid scheme fully discrete in time and space for solving the Navier-Stokes system. In the first step, the fully non-linear problem is discretized in space on a coarse grid with mesh-size $H$ and time step $k$. In the second step, the problem is discretized in space on a fine grid with mesh-size $h$ and the same time step, and linearized around the velocity $u_{H}$ computed in the first step. The two-grid strategy is motivated by the fact that under suitable assumptions, the contribution of $u_{H}$ to the error in the non-linear term, is measured in the $L^{2}$ norm in space and time, and thus has a higher-order than if it were measured in the $H^{1}$ norm in space. We present the following results: if $h=H^{2}=k$, then the global error of the two-grid algorithm is of the order of $h$, the same as would have been obtained if the non-linear problem had been solved directly on the fine grid.
\end{abstract}

Mathematics Subject Classification. 35Q30, 74S10, 76D05.

Received June 6, 2006. Revised December 12, 2006.

\section{INTRODUCTION}

The two-grid method is a general strategy for solving a non-linear Partial Differential Equation (PDE), depending or not in time, with solution $u$. In a first step, we discretize the fully non-linear PDE on a coarse grid of mesh-size $H$ and we compute an approximate solution $u_{H}$. Then, in a second step, we linearize the PDE around $u_{H}$ and we discretize the linearized problem on a fine grid of mesh-size $h$; let $u_{h}^{\text {lin }}$ be the corresponding solution. Then, under suitable assumptions, we can prove that if $h, H$ and the time step $k$ are well-chosen, the global error of the two-grid algorithm $\left\|u-u_{h}^{\text {lin }}\right\|$ has the same order as the error $\left\|u-u_{h}\right\|$ that would have been obtained if the non-linear problem had been directly discretized on the fine grid.

Two-grid discretizations have been widely applied to linear and non-linear elliptic boundary value problems: $\mathrm{Xu}$ in [19-21] has pioneered their development. These methods have been extended to the steady Navier-Stokes equations, $c f$. for instance the work of Layton in [12], Layton and Lenferink in [13] and Girault and Lions in [6]. Also, this method has been applied to the time-dependent Navier-Stokes problem, cf. Girault and Lions [7] in which they analyze a semi-discrete algorithm.

\footnotetext{
Keywords and phrases. Two-grid scheme, non-linear problem, incompressible flow, time and space discretizations, duality argument, "superconvergence".

1 Current address: Faculté des Sciences et de Génie Informatique, Université Saint-Esprit de Kaslik, B.P. 446 Jounieh, Liban. Laboratoire Jacques-Louis Lions, Université Pierre et Marie Curie (Paris 6), Boîte Courrier 187, 4, place Jussieu, 75252 Paris Cedex 05, France. abboud@ann.jussieu.fr

${ }^{2}$ Faculté des Sciences, Université Saint-Joseph, B.P. 11-514 Riad El Solh, Beyrouth 1107 2050, Liban.
}

(C) EDP Sciences, SMAI 2008 
The purpose of this article is to solve by a two-grid scheme, on a coarse grid and a fine grid, the non-stationary incompressible Navier-Stokes problem and to show that the two-grid algorithm's global error is similar to the error of the direct resolution of the non-linear problem on a fine grid.

Let $\Omega$ be a bounded domain of $\mathbb{R}^{2}$ with a polygonal boundary $\partial \Omega$ and let ]0,T[ be a given time-interval. Consider the following Navier-Stokes equations for an incompressible fluid, with $u$ the velocity and $p$ the pressure

$$
\left.\frac{\partial u}{\partial t}(x, t)-\nu \Delta u(x, t)+u(x, t) \cdot \nabla u(x, t)+\nabla p(x, t)=f(x, t) \text { in } \Omega \times\right] 0, T[,
$$

with the incompressibility condition

$$
\operatorname{div} u(x, t)=0 \text { in } \Omega \times] 0, T[,
$$

the homogeneous Dirichlet boundary condition

$$
u(x, t)=0 \text { on } \partial \Omega \times] 0, T[,
$$

and the initial condition

where the notation $u \cdot \nabla u$ means

$$
u(x, 0)=0 \text { in } \Omega
$$

$$
u \cdot \nabla u=\sum_{i=1}^{2} u_{i} \frac{\partial u}{\partial x_{i}}
$$

Setting $L_{0}^{2}(\Omega)=\left\{q \in L^{2}(\Omega) ; \int_{\Omega} q \mathrm{~d} x=0\right\}$ and assuming that $f$ belongs to $L^{2}\left(0, T ; H^{-1}(\Omega)^{2}\right)$, it is well-known that (1.1)-(1.2) has the following variational formulation in $] 0, T[$ :

Find $u(t) \in H_{0}^{1}(\Omega)^{2}$, such that in the sense of distributions on $] 0, T[$,

$$
\begin{gathered}
\forall v \in H_{0}^{1}(\Omega)^{2}, \frac{\mathrm{d}}{\mathrm{d} t}(u(t), v)+\nu(\nabla u(t), \nabla v)+(u(t) \cdot \nabla u(t), v)-(p(t), \operatorname{div} v)=\langle f(t), v\rangle, \\
\forall q \in L_{0}^{2}(\Omega),(q, \operatorname{div} u(t))=0,
\end{gathered}
$$

and

where $u(t)=u(x, t)$.

$$
u(0)=0,
$$

Furthermore, this problem has one and only one solution $u$ in $L^{\infty}\left(0, T ; L^{2}(\Omega)^{2}\right) \cap L^{2}\left(0, T ; H^{1}(\Omega)^{2}\right)$ and $p$ in the dual space of $W_{0}^{1,1}\left(0, T ; L_{0}^{2}(\Omega)\right)$ (see e.g. Ladyzenskaya in [11] and Lions in [14]).

In addition, we have the following regularity result.

Theorem 1.1. If $\Omega$ is convex and $f \in L^{2}\left(0, T ; L^{2}(\Omega)^{2}\right)$, then

$$
u \in L^{\infty}\left(0, T ; H^{1}(\Omega)^{2}\right) \cap L^{2}\left(0, T ; H^{2}(\Omega)^{2}\right) \text { and } p \in L^{2}\left(0, T ; H^{1}(\Omega)\right) .
$$

For discretizing (1.5)-(1.7), let $\eta>0$ be a discretization parameter in space and for each $\eta$, let $\mathcal{T}_{\eta}$ be a corresponding regular (or non-degenerate) family of triangulations of $\bar{\Omega}$, consisting of triangles such that any two triangles are either disjoint or share a vertex or an entire side. For an arbitrary triangle $\kappa$, we denote by $\eta_{\kappa}$ the diameter of $\kappa$ and by $\rho_{\kappa}$ the diameter of the circle inscribed in $\kappa$. Then $\eta$ denotes the maximum of $\eta_{\kappa}$ and we assume that $\mathcal{T}_{\eta}$ is regular in the sense of Ciarlet [5]: there exists a constant $\sigma$ independent of $\eta$ such that

$$
\sup _{\kappa \in \mathcal{I}_{\eta}} \frac{\eta_{\kappa}}{\rho_{\kappa}}=\sigma_{\kappa} \leq \sigma .
$$


Let $X_{\eta}$ and $M_{\eta}$ be a "stable" pair of finite-element spaces for discretizing the velocity $u$ and the pressure $p$, stable in the sense that it satisfies a uniform discrete inf-sup condition: there exists a constant $\beta^{\star} \geq 0$, independent of $\eta$, such that

$$
\forall q_{\eta} \in M_{\eta}, \sup _{v_{\eta} \in X_{\eta}} \frac{1}{\left|v_{\eta}\right|_{H^{1}(\Omega)}} \int_{\Omega} q_{\eta} \operatorname{div} v_{\eta} \mathrm{d} x \geq \beta^{\star}\left\|q_{\eta}\right\|_{L^{2}(\Omega)} .
$$

Let $\mathbb{P}_{\kappa}$ denote the space of polynomials with total degree less than or equal to $\kappa$. As the two-grid scheme is better adapted to finite-elements of low degree, we may choose for instance the "mini-element" (see Arnold et al. in [4]), where in each triangle $\kappa$, the pressure $p$ is a polynomial of $\mathbb{P}_{1}$ and each component of the velocity is the sum of a polynomial of $\mathbb{P}_{1}$ and a "bubble" function $b_{\kappa}$.

Denoting the vertices of $\kappa$ by $a_{i}, 1 \leq i \leq 3$, and its corresponding barycentric coordinate by $\lambda_{i}$, the basic bubble function $b_{\kappa}$ is the polynomial of degree three

$$
b_{\kappa}(x)=\lambda_{1}(x) \lambda_{2}(x) \lambda_{3}(x) .
$$

We observe that $b_{\kappa}(x)=0$ on $\partial \kappa$ and that $b_{\kappa}(x)>0$ on $\kappa$. The graph of $b_{\kappa}$ looks like a bulb attached to the boundary of $\kappa$, whence its name.

Therefore, the finite-element spaces are:

where

$$
\begin{aligned}
& X_{\eta}=\left\{v_{\eta} \in C^{0}(\bar{\Omega})^{2} ; \forall \kappa \in \mathcal{T}_{\eta}, v_{\left.\eta\right|_{\kappa}} \in \mathcal{P}(\kappa), v_{\left.\eta\right|_{\partial \Omega}}=0\right\}, \\
& M_{\eta}=\left\{q_{\eta} \in C^{0}(\bar{\Omega}) ; \forall \kappa \in \mathcal{T}_{\eta}, q_{\left.\eta\right|_{\kappa}} \in \mathbb{P}_{1}, \int_{\Omega} q_{\eta} \mathrm{d} x=0\right\},
\end{aligned}
$$

$$
\mathcal{P}(\kappa)=\left[\mathbb{P}_{1} \oplus \operatorname{Vect}\left(b_{\kappa}\right)\right]^{2} .
$$

There exists an approximation operator $P_{\eta} \in \mathcal{L}\left(H_{0}^{1}(\Omega)^{2} ; X_{\eta}\right)$ such that (see Girault and Raviart in [8]):

$$
\forall v \in H_{0}^{1}(\Omega)^{2}, \quad \forall q_{\eta} \in M_{\eta}, \quad \int_{\Omega} q_{\eta} \operatorname{div}\left(P_{\eta}(v)-v\right) \mathrm{d} x=0,
$$

and for $k=0$ or 1 ,

$$
\forall v \in\left[H^{1+k}(\Omega) \cap H_{0}^{1}(\Omega)\right]^{2}, \quad\left\|P_{\eta}(v)-v\right\|_{L^{2}(\Omega)} \leq C \eta^{1+k}|v|_{H^{1+k}(\Omega)},
$$

and for all $r \geq 2, k=0$ or 1 ,

$$
\forall v \in\left[W^{1+k, r}(\Omega) \cap H_{0}^{1}(\Omega)\right]^{2}, \quad\left|P_{\eta}(v)-v\right|_{W^{1, r}(\Omega)} \leq C_{r} \eta^{k}|v|_{W^{1+k, r}(\Omega)} .
$$

In addition, as $M_{\eta}$ contains all polynomials of degree one, there exists an operator $r_{\eta} \in \mathcal{L}\left(L_{0}^{2}(\Omega) ; M_{\eta}\right)$, such that for any real number $s \in[0,2]$,

$$
\forall q \in H^{s}(\Omega) \cap L_{0}^{2}(\Omega),\left\|r_{\eta}(q)-q\right\|_{L^{2}(\Omega)} \leq C \eta^{s}|q|_{H^{s}(\Omega)} .
$$

To discretize in time, we divide the interval $[0, T]$ into $N$ subintervals of equal length $k=\frac{T}{N}$, with grid-points $t^{n}=n k, 0 \leq n \leq N$.

With these spaces, we propose the following two-grid scheme for discretizing (1.5)-(1.7). We use two regular nested triangulations of $\bar{\Omega}$ : a coarse triangulation $\mathcal{T}_{H}$ and a fine one $\mathcal{T}_{h}$, that for practical purposes, is a refinement of $\mathcal{T}_{H}$. In that case, the interpolation/projection procedure is easy.

On each of these, we define the same stable pair of finite-element spaces, $\left(X_{H}, M_{H}\right)$ and $\left(X_{h}, M_{h}\right)$ such that $X_{H} \subset X_{h}$ and $M_{H} \subset M_{h}$. At each time step, we solve (1.18)-(1.19) and (1.20)-(1.21) below. The two-grid algorithm reads: 
- Step one (non-linear problem on coarse grid). Knowing $u_{H}^{n}$, find $\left(u_{H}^{n+1}, p_{H}^{n+1}\right)$ with values in $X_{H} \times M_{H}$, solution of

$$
\begin{gathered}
\forall v_{H} \in X_{H}, 1 k\left(u_{H}^{n+1}-u_{H}^{n}, v_{H}\right)+\nu\left(\nabla u_{H}^{n+1}, \nabla v_{H}\right)+\left(u_{H}^{n+1} \cdot \nabla u_{H}^{n+1}, v_{H}\right)+\frac{1}{2}\left(\operatorname{div} u_{H}^{n+1}, u_{H}^{n+1} \cdot v_{H}\right) \\
-\left(p_{H}^{n+1}, \operatorname{div} v_{H}\right)=\left\langle f^{n+1}, v_{H}\right\rangle \\
\forall q_{H} \in M_{H},\left(q_{H}, \operatorname{div} u_{H}^{n+1}\right)=0 .
\end{gathered}
$$

- Step two (linearized problem on fine grid). Knowing $\left(u_{H}^{n+1}, p_{H}^{n+1}\right)$, find $\left(u_{h}^{n+1}, p_{h}^{n+1}\right)$ with values in $X_{h} \times M_{h}$ solution of

$$
\begin{gathered}
\forall v_{h} \in X_{h}, \frac{1}{k}\left(u_{h}^{n+1}-u_{h}^{n}, v_{h}\right)+\nu\left(\nabla u_{h}^{n+1}, \nabla v_{h}\right)+\left(u_{H}^{n+1} \cdot \nabla u_{h}^{n+1}, v_{h}\right)-\left(p_{h}^{n+1}, \operatorname{div} v_{h}\right) \\
\forall q_{h} \in M_{h},\left(q_{h}, \operatorname{div} u_{h}^{n+1}\right)=0 .
\end{gathered}
$$

By assumption, $u_{h}^{0}=0$. Moreover, at the step time $n+1$, in (1.18), $u_{H}^{n}$ is in fact a restriction on the coarse grid of $u_{h}^{n}$ that has just been computed:

where $\mathcal{R}$ is a suitable restriction from $X_{h}$ into $X_{H}$.

$$
u_{H}^{n}=\mathcal{R}\left(u_{h}^{n}\right)
$$

The pressure $p_{h}^{n+1}$ is dissociated from $u_{h}^{n+1}$ by a decoupling algorithm starting with an extension of $p_{H}^{n+1}$ to the fine grid.

In both (1.18) and (1.20), $f^{n+1}$ is a suitable approximation of $f$ at time $t^{n+1}$. The purpose of this two-grid algorithm is to reduce the time of computation for both velocity and pressure.

In the sequel, we shall take $k$ of the order of $H^{2}$ : there exist two constants $\alpha_{1}$ and $\alpha_{2}>0$ that do not depend on $H$ and $k$ such that

$$
\alpha_{1} H^{2} \leq k \leq \alpha_{2} H^{2}
$$

In what follows, all constants are independent of the space steps $h, H$ and the time step $k$.

Remark 1.2. To simplify the error analysis, the convection term in (1.18) is stabilized so that it is antisymmetric. But often in practice, it is not stabilized. We refer to [7] for the numerical analysis of a semi-discrete scheme that is not stabilized. We note that in that case, the data $f$ must satisfy a condition in order to have the stability of the scheme.

Remark 1.3. One can also linearize the first step, without the anti-symmetric term, by taking the non-linear term at time $n$ (instead of $n+1$ ). This requires a condition CFL, but as $k \ll H$, this condition is generally satisfied. Once we stabilize the scheme, this condition CFL is not required.

Remark 1.4. This is an example in which both equations use the same time step and are both of order one with respect to time. A more elaborate idea for Step two would be to use a scheme of second-order in time with the same time step, or some time-splitting scheme of order one.

The remainder of this article is organized as follows: In Section 2, we present some conventions and notations that will be used throughout the article. In Section 3, we present a first error estimate for the fully-discrete Step one then in Section 4 we establish a duality argument based on the backward semi-discrete Stokes system and we derive some uniform bounds that allow us to prove the Stokes problem's error estimate in $L^{2}(\Omega \times] 0, T[)^{2}$, then we apply it to the Navier-Stokes problem. We also prove a "superconvergence" result for the non-linear part. The pressure is estimated in Section 5 and the error estimation for the solution of Step two is studied in Section 6. Finally, in Section 7, we confirm these results numerically.

Some of these results have been announced in [1]. 


\section{Preliminaries}

To begin with, we present some conventions and notations that will be used throughout the article. As usual, for handling time-dependent problems, it is convenient to consider functions defined on a time interval $] a, b[$ with values in a functional space, say $X$ (cf. Lions and Magenes [15]). More precisely, let $\|\cdot\|_{X}$ denote the norm of $X$; then for any $r, 1 \leq r \leq \infty$, we define

$$
L^{r}(a, b ; X)=\{f \text { measurable in }] a, b\left[; \int_{a}^{b}\|f(t)\|_{X}^{r} \mathrm{~d} t<\infty\right\}
$$

equipped with the norm

$$
\|f\|_{L^{r}(a, b ; X)}=\left(\int_{a}^{b}\|f(t)\|_{X}^{r} \mathrm{~d} t\right)^{1 / r}
$$

with the usual modifications if $r=\infty$. It is a Banach space if $X$ is a Banach space.

Let $\left(k_{1}, k_{2}\right)$ denote a pair of non-negative integers, set $|k|=k_{1}+k_{2}$ and define the partial derivative $\partial^{k}$ by $\partial^{k} v=\frac{\partial^{|k|} v}{\partial x_{1}^{k_{1}} \partial x_{2}^{k_{2}}}$.

Here $X$ is usually a Sobolev space, such as ( $c f$. Adams [3] or Nečas [16]): for any non-negative integer $m$ and number $r \geq 1$,

$$
W^{m, r}(\Omega)=\left\{v \in L^{r}(\Omega) ; \partial^{k} v \in L^{r}(\Omega), \forall|k| \leq m\right\}
$$

This space is equipped with the seminorm $|v|_{W^{m, r}(\Omega)}=\left[\sum_{|k|=m} \int_{\Omega}\left|\partial^{k} v\right|^{r} \mathrm{~d} x\right]^{1 / r}$, and is a Banach space for the norm $\|v\|_{W^{m, r}(\Omega)}=\left[\sum_{0 \leq|k| \leq m}|v|_{W^{k, r}(\Omega)}^{r}\right]^{1 / r}$, with the usual extension when $r=\infty$.

When $r=2$, this space is the Hilbert space $H^{m}(\Omega)$. In particular, the scalar product of $L^{2}(\Omega)$ is denoted by $(\cdot, \cdot)$.

Similarly, $L^{2}\left(a, b ; H^{m}(\Omega)\right)$ is a Hilbert space and in particular $L^{2}\left(a, b ; L^{2}(\Omega)\right)$ coincides with $L^{2}(\Omega \times] a, b[)$. The definitions of these spaces are extended straightforwardly to vectors, with the same notation, but with the following modification for the norms in the non-Hilbert case. Let $u=\left(u_{1}, u_{2}\right)$; then we set

$$
\|u\|_{L^{r}(\Omega)}=\left[\int_{\Omega}\|u(x)\|^{r} \mathrm{~d} x\right]^{1 / r}
$$

where $\|\cdot\|$ denotes the Euclidean vector norm.

For functions that vanish on the boundary, we recall the inequalities of Sobolev imbeddings in two dimensions: for each $r \in\left[2, \infty\left[\right.\right.$, there exits a constant $S_{r}$ such that

$$
\forall v \in H_{0}^{1}(\Omega),\|v\|_{L^{r}(\Omega)} \leq S_{r}|v|_{H^{1}(\Omega)},
$$

where

$$
|v|_{H^{1}(\Omega)}=\|\nabla v\|_{L^{2}(\Omega)} .
$$

When $r=2$, (2.1) reduces to Poincaré's inequality and $S_{2}$ is Poincaré's constant. The case $r=\infty$ is excluded and is replaced by: For any $r>2$, there exists a constant $M_{r}$ such that

$$
\forall v \in W_{0}^{1, r}(\Omega),\|v\|_{L^{\infty}(\Omega)} \leq M_{r}|v|_{W^{1, r}(\Omega)} .
$$

We have also in dimension 2 ,

$$
\|g\|_{L^{4}(\Omega)} \leq 2^{1 / 4}\|g\|_{L^{2}(\Omega)}^{1 / 2}\|\nabla g\|_{L^{2}(\Omega)}^{1 / 2}
$$


Also, we recall the spaces we introduced at the beginning:

$$
V=\left\{v \in H_{0}^{1}(\Omega)^{2} ; \operatorname{div} v=0 \text { in } \Omega\right\}, L_{0}^{2}(\Omega)=\left\{q \in L^{2}(\Omega) ; \int_{\Omega} q \mathrm{~d} x=0\right\}
$$

and the orthogonal complement of $V$ in $H_{0}^{1}(\Omega)^{2}$ :

$$
V^{\perp}=\left\{v \in H_{0}^{1}(\Omega)^{2} ; \forall w \in V,(\nabla v, \nabla w)=0\right\} .
$$

\section{Error estimates for the solution of Step one}

The results in this paragraph are written for the non-linear scheme (1.18)-(1.19).

To simplify, we denote by $\eta$ the mesh parameter. The first result, stated in Lemma 3.2, is a standard error estimate. We give the proof for the sake of completeness.

Remark 3.1. In what follows, when we have, for example,

$$
\left\|v_{h}^{m}\right\|_{L^{2}(\Omega)}^{2}+\sum_{n=0}^{m-1}\left\|v_{h}^{n+1}-v_{h}^{n}\right\|_{L^{2}(\Omega)}^{2}+\nu \sum_{n=0}^{m-1} k\left|v_{h}^{n+1}\right|_{H^{1}(\Omega)}^{2} \leq C_{1}+C_{2} \sum_{n=0}^{m-1} k\left\|v_{h}^{n+1}\right\|_{L^{2}(\Omega)}^{2},
$$

we write,

then

$$
\left\|v_{h}^{m}\right\|_{L^{2}(\Omega)} \leq\left\|v_{h}^{m}-v_{h}^{m-1}\right\|_{L^{2}(\Omega)}+\left\|v_{h}^{m-1}\right\|_{L^{2}(\Omega)},
$$

$$
C_{2} k\left\|v_{h}^{m}\right\|_{L^{2}(\Omega)}^{2} \leq 2 C_{2} k\left\|v_{h}^{m}-v_{h}^{m-1}\right\|_{L^{2}(\Omega)}^{2}+2 C_{2} k\left\|v_{h}^{m-1}\right\|_{L^{2}(\Omega)}^{2} .
$$

We suppose $k$ sufficiently small such that $2 C_{2} k \leq 1$ (for example). Then we obtain

$$
\left\|v_{h}^{m}\right\|_{L^{2}(\Omega)}^{2}+\sum_{n=0}^{m-2}\left\|v_{h}^{n+1}-v_{h}^{n}\right\|_{L^{2}(\Omega)}^{2}+\nu \sum_{n=0}^{m-1} k\left|v_{h}^{n+1}\right|_{H^{1}(\Omega)}^{2} \leq C_{1}+3 C_{2} \sum_{n=1}^{m-1} k\left\|v_{h}^{n}\right\|_{L^{2}(\Omega)}^{2},
$$

and we apply the classic Gronwall's lemma. (We can also keep the term $\left\|v_{h}^{m}-v_{h}^{m-1}\right\|_{L^{2}(\Omega)}^{2}$ multiplied by a factor $\alpha<1$.)

Lemma 3.2. Let $X_{\eta}$ and $M_{\eta}$ be defined by (1.11) and (1.12) and approximate $f^{n+1}$ by the average defined almost everywhere in $\Omega$ as follows:

$$
f^{n+1}(x)=\frac{1}{k} \int_{t^{n}}^{t^{n+1}} f(x, t) \mathrm{d} t, \text { a.e } x \in \Omega .
$$

At each time step, (1.18)-(1.19) has a solution $u_{\eta}^{n+1}$ and this solution is unique if $k$ is sufficiently small.

Under the assumptions $u \in L^{\infty}\left(0, T ; H^{1}(\Omega)^{2}\right) \cap L^{2}\left(0, T ; H^{2}(\Omega)^{2}\right), u^{\prime} \in L^{2}\left(0, T ; H^{1}(\Omega)^{2}\right)$ and $p \in L^{2}(0, T$; $H^{1}(\Omega)$ ), there exist constants $C$ and $k_{0}>0$, independent of $\eta$ and $k$ such that each solution satisfies, for $k \leq k_{0}$ :

$$
\begin{aligned}
\sup _{0 \leq n \leq N}\left\|u_{\eta}^{n}-u\left(t^{n}\right)\right\|_{L^{2}(\Omega)}+\left(\sum_{n=0}^{N-1} \|\right. & \left.\left(u_{\eta}^{n+1}-u\left(t^{n+1}\right)\right)-\left(u_{\eta}^{n}-u\left(t^{n}\right)\right) \|_{L^{2}(\Omega)}^{2}\right)^{1 / 2} \\
& +\sqrt{\nu}\left(\sum_{n=0}^{N-1} k\left|u_{\eta}^{n+1}-u\left(t^{n+1}\right)\right|_{H^{1}(\Omega)}^{2}\right)^{1 / 2} \leq C(f, u, p, \nu, T)(\eta+k) .
\end{aligned}
$$


Proof. Noting that the approximation operator $P_{\eta}$ defined in [8] (Chap. 1, pp. 101-102) satisfies $\left(P_{\eta}(u)\right)^{\prime}=P_{\eta}\left(u^{\prime}\right)$, we choose the test function $v_{\eta}^{n+1}=u_{\eta}^{n+1}-P_{\eta} u\left(t^{n+1}\right)$ in (1.1) then integrate it over $\left[t^{n}, t^{n+1}\right]$, substract (1.18), insert $P_{\eta} u\left(t^{n+1}\right)$, multiply the result by the time step $k$ and sum it over $n=0, \ldots, m-1$. We obtain:

$$
\begin{aligned}
& \frac{1}{2}\left(\left\|v_{\eta}^{m}\right\|_{L^{2}(\Omega)}^{2}-\left\|v_{\eta}^{0}\right\|_{L^{2}(\Omega)}^{2}+\sum_{n=0}^{m-1}\left\|v_{\eta}^{n+1}-v_{\eta}^{n}\right\|_{L^{2}(\Omega)}^{2}\right)+\nu \sum_{n=0}^{m-1} k\left\|\nabla v_{\eta}^{n+1}\right\|_{L^{2}(\Omega)}^{2}= \\
& \sum_{n=0}^{m-1}\left\{\left(\left(u\left(t^{n+1}\right)-P_{\eta} u\left(t^{n+1}\right)\right)-\left(u\left(t^{n}\right)-P_{\eta} u\left(t^{n}\right)\right), v_{\eta}^{n+1}\right)+\nu \int_{t^{n}}^{t^{n+1}}\left(\nabla\left(u(t)-P_{\eta} u\left(t^{n+1}\right)\right), \nabla v_{\eta}^{n+1}\right) \mathrm{d} t\right. \\
& \quad+\int_{t^{n}}^{t^{n+1}}\left(\left(u(t) \cdot \nabla u(t)-u_{\eta}^{n+1} \cdot \nabla u_{\eta}^{n+1}\right)+\frac{1}{2}\left(\operatorname{div} u(t) u(t)-\operatorname{div} u_{\eta}^{n+1} u_{\eta}^{n+1}\right), v_{\eta}^{n+1}\right) \mathrm{d} t \\
& \left.-\int_{t^{n}}^{t^{n+1}}\left(p(t)-r_{\eta} p(t), \operatorname{div} v_{\eta}^{n+1}\right) \mathrm{d} t\right\} .
\end{aligned}
$$

Let us study the terms of the right hand side of (3.3). The non-linear term is treated like follows:

$$
\begin{aligned}
u(t) \cdot \nabla u(t)-u_{\eta}^{n+1} \cdot \nabla u_{\eta}^{n+1}= & \left(u(t)-P_{\eta} u\left(t^{n+1}\right)\right) \cdot \nabla u(t)-v_{\eta}^{n+1} \cdot \nabla P_{\eta} u\left(t^{n+1}\right) \\
& +P_{\eta} u\left(t^{n+1}\right) \cdot \nabla\left(u(t)-P_{\eta} u\left(t^{n+1}\right)\right)+u_{\eta}^{n+1} \cdot \nabla\left(P_{\eta} u\left(t^{n+1}\right)-u_{\eta}^{n+1}\right)
\end{aligned}
$$

and the term corresponding to the divergence is treated similarly.

The first term is bounded as follows: For any $\varepsilon_{1}>0$,

$$
\left|\sum_{n=0}^{m-1}\left(\left(u\left(t^{n+1}\right)-P_{\eta} u\left(t^{n+1}\right)\right)-\left(u\left(t^{n}\right)-P_{\eta} u\left(t^{n}\right)\right), v_{\eta}^{n+1}\right)\right| \leq \frac{C^{2}}{2 \varepsilon_{1}}\left\|u^{\prime}\right\|_{L^{2}\left(0, T ; H^{1}(\Omega)^{2}\right)}^{2} \eta^{2}+\frac{\varepsilon_{1} S_{2}}{2} \sum_{n=0}^{m-1} k\left|v_{\eta}^{n+1}\right|_{H^{1}(\Omega)}^{2},
$$

where $S_{2}$ is the constant of Poincaré's inequality.

To study the second term, we insert $P_{\eta} u(t)$ and we obtain two terms: For any $\varepsilon_{2}>0$, the first one is bounded as follows:

$$
\begin{aligned}
\left|\nu \sum_{n=0}^{m-1} \int_{t^{n}}^{t^{n+1}}\left(\nabla\left(u(t)-P_{\eta} u(t)\right), \nabla v_{\eta}^{n+1}\right) \mathrm{d} t\right| & \leq \frac{\nu}{2}\left\{\frac{1}{\varepsilon_{2}} \sum_{n=0}^{m-1} \int_{t^{n}}^{t^{n+1}}\left|u(t)-P_{\eta} u(t)\right|_{H^{1}(\Omega)}^{2} \mathrm{~d} t+\varepsilon_{2} \sum_{n=0}^{m-1} k\left|v_{\eta}^{n+1}\right|_{H^{1}(\Omega)}^{2}\right\} \\
& \leq \frac{\nu}{2}\left\{\frac{C}{\varepsilon_{2}}\|u\|_{L^{2}\left(0, T ; H^{2}(\Omega)^{2}\right)}^{2} \eta^{2}+\varepsilon_{2} \sum_{n=0}^{m-1} k\left|v_{\eta}^{n+1}\right|_{H^{1}(\Omega)}^{2}\right\}
\end{aligned}
$$

and the second one as follows: Knowing that

$$
\int_{t^{n}}^{t^{n+1}} P_{\eta}\left(u(t)-u\left(t^{n+1}\right)\right) \mathrm{d} t=\int_{t^{n}}^{t^{n+1}} P_{\eta} u^{\prime}(\tau)\left(\tau-t^{n}\right) \mathrm{d} \tau
$$

we have, for any $\varepsilon_{3}>0$,

$$
\left|\nu \sum_{n=0}^{m-1} \int_{t^{n}}^{t^{n+1}}\left(\nabla P_{\eta}\left(u(t)-u\left(t^{n+1}\right)\right), \nabla v_{\eta}^{n+1}\right) \mathrm{d} t\right| \leq \frac{\nu C}{2 \sqrt{3} \varepsilon_{3}}\left\|u^{\prime}\right\|_{L^{2}\left(0, T ; H^{1}(\Omega)^{2}\right)}^{2} k^{2}+\frac{\nu}{2 \sqrt{3}} \varepsilon_{3} \sum_{n=0}^{m-1} k\left|v_{\eta}^{n+1}\right|_{H^{1}(\Omega)}^{2} .
$$


For the pressure contribution, we have, for any $\varepsilon_{4}>0$,

$$
\begin{aligned}
\left|\sum_{n=0}^{m-1}-\int_{t^{n}}^{t^{n+1}}\left(p(t)-r_{\eta} p(t), \operatorname{div} v_{\eta}^{n+1}\right) \mathrm{d} t\right| & \leq\left(\sum_{n=0}^{m-1} \int_{t^{n}}^{t^{n+1}}\left\|p(t)-r_{\eta} p(t)\right\|_{L^{2}(\Omega)}^{2} \mathrm{~d} t\right)^{1 / 2}\left(\sum_{n=0}^{m-1} k\left|v_{\eta}^{n+1}\right|_{H^{1}(\Omega)}^{2}\right)^{1 / 2} \\
& \leq \frac{C}{2 \varepsilon_{4}}\|p\|_{L^{2}\left(0, T ; H^{1}(\Omega)\right)}^{2} \eta^{2}+\frac{\varepsilon_{4}}{2} \sum_{n=0}^{m-1} k\left|v_{\eta}^{n+1}\right|_{H^{1}(\Omega)}^{2} .
\end{aligned}
$$

Now, we consider the non-linear terms. Applying (2.1) and (2.4) and setting

$$
C_{1}=\|u\|_{L^{\infty}\left(0, T ; H^{1}(\Omega)^{2}\right)},
$$

we have, for any $\varepsilon_{5}$ and $\varepsilon_{6}>0$,

$$
\left|\sum_{n=0}^{m-1} \int_{t^{n}}^{t^{n+1}}\left(\left(u(t)-P_{\eta} u(t)\right) \cdot \nabla u(t), v_{\eta}^{n+1}\right) \mathrm{d} t\right| \leq \frac{C_{1} S_{4}^{2}}{2}\left\{\frac{C}{\varepsilon_{5}}\|u\|_{L^{2}\left(0, T ; H^{2}(\Omega)^{2}\right)}^{2} \eta^{2}+\varepsilon_{5} \sum_{n=0}^{m-1} k\left|v_{\eta}^{n+1}\right|_{H^{1}(\Omega)}^{2}\right\},
$$

and

$$
\left|\sum_{n=0}^{m-1} \int_{t^{n}}^{t^{n+1}}\left(P_{\eta}\left(u(t)-u\left(t^{n+1}\right)\right) \cdot \nabla u(t), v_{\eta}^{n+1}\right) \mathrm{d} t\right| \leq \frac{C_{1} S_{4}^{2}}{2 \sqrt{3}}\left\{\frac{k^{2}}{\varepsilon_{6}}\left\|u^{\prime}\right\|_{L^{2}\left(0, T ; H^{1}(\Omega)^{2}\right)}^{2}+\varepsilon_{6} \sum_{n=0}^{m-1} k\left|v_{\eta}^{n+1}\right|_{H^{1}(\Omega)}^{2}\right\} .
$$

The corresponding divergence terms are bounded as follows: For any $\varepsilon_{7}$ and $\varepsilon_{8}>0$,

$$
\left|\frac{1}{2} \sum_{n=0}^{m-1} \int_{t^{n}}^{t^{n+1}}\left(\operatorname{div}\left(u(t)-P_{\eta} u(t)\right) \cdot u(t), v_{\eta}^{n+1}\right) \mathrm{d} t\right| \leq \frac{S_{4}^{2} C_{1}}{4}\left\{\frac{C}{\varepsilon_{7}}\|u\|_{L^{2}\left(0, T ; H^{2}(\Omega)^{2}\right)}^{2} \eta^{2}+\varepsilon_{7} \sum_{n=0}^{m-1} k\left|v_{\eta}^{n+1}\right|_{H^{1}(\Omega)}^{2}\right\},
$$

and

$$
\left|\frac{1}{2} \sum_{n=0}^{m-1} \int_{t^{n}}^{t^{n+1}}\left(\operatorname{div} P_{\eta}\left(u(t)-u\left(t^{n+1}\right)\right) \cdot u(t), v_{\eta}^{n+1}\right) \mathrm{d} t\right| \leq \frac{S_{4}^{2} C_{1}}{4 \sqrt{3}}\left\{\frac{k^{2}}{\varepsilon_{8}}\left\|u^{\prime}\right\|_{L^{2}\left(0, T ; H^{1}(\Omega)^{2}\right)}^{2}+\varepsilon_{8} \sum_{n=0}^{m-1} k\left|v_{\eta}^{n+1}\right|_{H^{1}(\Omega)}^{2}\right\} .
$$

Setting $C_{2}=\left\|P_{\eta} u\right\|_{L^{\infty}\left(0, T ; H^{1}(\Omega)^{2}\right)}$, we also have, for any $\varepsilon_{9}$ and $\varepsilon_{10}>0$,

$$
\left|-\sum_{n=0}^{m-1} \int_{t^{n}}^{t^{n+1}}\left(v_{\eta}^{n+1} \cdot \nabla P_{\eta} u\left(t^{n+1}\right), v_{\eta}^{n+1}\right) \mathrm{d} t\right| \leq \frac{2^{1 / 2} C_{1}}{2}\left\{\varepsilon_{9} \sum_{n=0}^{m-1} k\left|v_{\eta}^{n+1}\right|_{H^{1}(\Omega)}^{2}+\frac{1}{\varepsilon_{9}} \sum_{n=0}^{m-1} k\left\|v_{\eta}^{n+1}\right\|_{L^{2}(\Omega)}^{2}\right\},
$$

and

$$
\begin{aligned}
\left|-\frac{1}{2} \sum_{n=0}^{m-1} \int_{t^{n}}^{t^{n+1}}\left(\operatorname{div} v_{\eta}^{n+1} \cdot P_{\eta} u\left(t^{n+1}\right), v_{\eta}^{n+1}\right) \mathrm{d} t\right| \leq \frac{2^{1 / 4} S_{4} C_{2}}{4}\left\{\frac{1}{\varepsilon_{10}} \sum_{n=0}^{m-1} k\left|v_{\eta}^{n+1}\right|_{H^{1}(\Omega)}^{2}\right. & \\
& \left.+\frac{\varepsilon_{10}}{2} \sum_{n=0}^{m-1} k\left(\delta\left|v_{\eta}^{n+1}\right|_{H^{1}(\Omega)}^{2}+\frac{1}{\delta}\left\|v_{\eta}^{n+1}\right\|_{L^{2}(\Omega)}^{2}\right)\right\} .
\end{aligned}
$$

The two final terms are split as follows: For any $\varepsilon_{11}>0$,

$\left|\sum_{n=0}^{m-1} \int_{t^{n}}^{t^{n+1}}\left(P_{\eta} u\left(t^{n+1}\right) \cdot \nabla\left(u(t)-P_{\eta} u(t)\right), v_{\eta}^{n+1}\right) \mathrm{d} t\right| \leq \frac{S_{4}^{2} C_{2}}{2}\left\{\frac{C}{\varepsilon_{11}}\|u\|_{L^{2}\left(0, T ; H^{2}(\Omega)^{2}\right)}^{2} \eta^{2}+\varepsilon_{11} \sum_{n=0}^{m-1} k\left|v_{\eta}^{n+1}\right|_{H^{1}(\Omega)}^{2}\right\}$, 
with the divergence contribution: For any $\varepsilon_{12}$ and $\varepsilon_{13}>0$,

$$
\begin{array}{r}
\left|\frac{1}{2} \sum_{n=0}^{m-1} \int_{t^{n}}^{t^{n+1}}\left(\operatorname{div}\left(P_{\eta} u\left(t^{n+1}\right)\right)\left(u(t)-P_{\eta} u(t)\right), v_{\eta}^{n+1}\right) \mathrm{d} t\right| \leq \\
\frac{S_{4}^{2} C_{1}}{2}\left\{\frac{C}{\varepsilon_{12}}\|u\|_{L^{2}\left(0, T ; H^{2}(\Omega)^{2}\right)}^{2} \eta^{4}+\varepsilon_{12} \sum_{n=0}^{m-1} k\left|v_{\eta}^{n+1}\right|_{H^{1}(\Omega)}^{2}\right\}
\end{array}
$$

and

$$
\begin{aligned}
\left|\sum_{n=0}^{m-1} \int_{t^{n}}^{t^{n+1}}\left(P_{\eta} u\left(t^{n+1}\right) \cdot \nabla P_{\eta}\left(u(t)-u\left(t^{n+1}\right)\right), v_{\eta}^{n+1}\right) \mathrm{d} t\right| & \leq \\
& \frac{S_{4}^{2} C_{2} C}{2 \sqrt{3}}\left\{\frac{k^{2}}{\varepsilon_{13}}\left\|u^{\prime}\right\|_{L^{2}\left(0, T ; H^{1}(\Omega)^{2}\right)}^{2}+\varepsilon_{13} \sum_{n=0}^{m-1} k\left|v_{\eta}^{n+1}\right|_{H^{1}(\Omega)}^{2}\right\}
\end{aligned}
$$

and also the divergence contribution: For any $\varepsilon_{14}>0$,

$$
\begin{array}{r}
\left|\frac{1}{2} \sum_{n=0}^{m-1} \int_{t^{n}}^{t^{n+1}}\left(\operatorname{div}\left(P_{\eta} u\left(t^{n+1}\right)\right) P_{\eta}\left(u(t)-u\left(t^{n+1}\right)\right), v_{\eta}^{n+1}\right) \mathrm{d} t\right| \\
\frac{S_{4}^{2} C_{2} C}{4 \sqrt{3}}\left\{\frac{k^{2}}{\varepsilon_{14}}\left\|u^{\prime}\right\|_{L^{2}\left(0, T ; H^{1}(\Omega)^{2}\right)}^{2}+\varepsilon_{14} \sum_{n=0}^{m-1} k\left|v_{\eta}^{n+1}\right|_{H^{1}(\Omega)}^{2}\right\}
\end{array}
$$

The last term in (3.4) vanishes with $-\frac{1}{2} \operatorname{div} u_{\eta}^{n+1} \cdot\left(P_{\eta} u\left(t^{n+1}\right)-u_{\eta}^{n+1}\right)$.

After a suitable choice of $\varepsilon_{i}$ and $\delta,(3.3)$ becomes

$$
\frac{1}{2}\left\|v_{\eta}^{m}\right\|_{L^{2}(\Omega)}^{2}+\frac{1}{2} \sum_{n=0}^{m-1}\left\|v_{\eta}^{n+1}-v_{\eta}^{n}\right\|_{L^{2}(\Omega)}^{2}+\frac{\nu}{2} \sum_{n=0}^{m-1} k\left|v_{\eta}^{n+1}\right|_{H^{1}(\Omega)}^{2} \leq C_{\star}+C \sum_{n=0}^{m-1} k\left\|v_{\eta}^{n+1}\right\|_{L^{2}(\Omega)}^{2}
$$

where $C_{\star}=\alpha \eta^{2}+\beta k^{2}, \alpha$ and $\beta$ are constants that depend on $u, p, \nu$, but do not depend on $\eta$ and $k$.

Then after applying Gronwall's lemma and for $k$ sufficiently small, the result follows from this inequality:

$$
\begin{gathered}
\sup _{0 \leq n \leq N}\left\|u_{\eta}^{n}-P_{\eta} u\left(t^{n}\right)\right\|_{L^{2}(\Omega)}+\left(\sum_{n=0}^{N-1}\left\|\left(u_{\eta}^{n+1}-P_{\eta} u\left(t^{n+1}\right)\right)-\left(u_{\eta}^{n}-P_{\eta} u\left(t^{n}\right)\right)\right\|_{L^{2}(\Omega)}^{2}\right)^{1 / 2} \\
+\sqrt{\nu}\left(\sum_{n=0}^{N-1} k\left|u_{\eta}^{n+1}-P_{\eta} u\left(t^{n+1}\right)\right|_{H^{1}(\Omega)}^{2}\right)^{1 / 2} \leq C(\eta+k) .
\end{gathered}
$$

Finally, (3.2) follows by applying a triangular inequality and the $P_{\eta}$ 's properties.

The next property of the solution of (1.18)-(1.19) is an easy consequence of Lemma 3.2.

Corollary 3.3. In addition to the assumptions of Lemma 3.2, we assume that there exists a constant $\alpha>0$ independent of $\eta$ and $k$, such that $k \geq \alpha \eta^{2}$. Then, there exists a constant $C$ independent of $\eta$ and $k$ such that

$$
\sup _{n}\left|u_{\eta}^{n}\right|_{H^{1}(\Omega)} \leq C \text {. }
$$


Proof. We have

$$
\left(\sum_{n=0}^{N-1} k\left|u_{\eta}^{n+1}-u\left(t^{n+1}\right)\right|_{H^{1}(\Omega)}^{2}\right)^{1 / 2} \leq C(\eta+k)
$$

which implies that

$$
\left|u_{\eta}^{n}-u\left(t^{n}\right)\right|_{H^{1}(\Omega)}^{2} \leq \frac{C(\eta+k)^{2}}{k} \leq C\left(\frac{\eta^{2}}{k}+k\right) \leq C, \quad 0 \leq n \leq N
$$

Then

$$
\left|u_{\eta}^{n}\right|_{H^{1}(\Omega)} \leq\left|u_{\eta}^{n}-u\left(t^{n}\right)\right|_{H^{1}(\Omega)}+\left|u\left(t^{n}\right)\right|_{H^{1}(\Omega)} \leq C .
$$

Remark 3.4. We suppose that there exist two constants $\alpha$ and $\gamma>0$ that do not depend on $\eta$ and $k$ such that

$$
\alpha \eta^{2} \leq k \leq \gamma \eta^{2}
$$

which means that $k$ is of the same order of $\eta^{2}$.

\section{Some ERror estimates for the Stokes Problem}

The error estimate of order two in $L^{2}(\Omega \times] 0, T[)^{2}$, that will be established in the next section, is based on a duality argument for the transient Stokes problem:

$$
\begin{gathered}
\left.\frac{\partial v}{\partial t}(x, t)-\nu \Delta v(x, t)+\nabla q(x, t)=g(x, t) \text { in } \Omega \times\right] 0, T[, \\
\operatorname{div} v(x, t)=0 \text { in } \Omega \times] 0, T[, \\
v(x, t)=0 \text { on } \partial \Omega \times] 0, T[, \\
v(x, 0)=0 \text { in } \Omega .
\end{gathered}
$$

Theorem 4.1. This problem has a unique solution $(v, q)$. We assume that $g \in L^{2}(\Omega \times] 0, T[)^{2}$. Then

$$
v \in L^{2}\left(0, T ; W^{2,4 / 3}(\Omega)^{2}\right) \cap L^{\infty}\left(0, T ; H^{1}(\Omega)^{2}\right), v^{\prime} \in L^{2}(\Omega \times] 0, T[)^{2} \text { and } q \in L^{2}\left(0, T ; W^{1,4 / 3}(\Omega)\right) .
$$

If $\Omega$ is convex, then $(v, q) \in L^{2}\left(0, T ; H^{2}(\Omega)^{2}\right) \times L^{2}\left(0, T ; H^{1}(\Omega)\right)$. Finally, without convexity assumption, if $g \in H^{1}\left(0, T ; H^{-1}(\Omega)^{2}\right)$ and $g(0) \in L^{2}(\Omega)^{2}$, then $v^{\prime} \in L^{\infty}\left(0, T ; L^{2}(\Omega)^{2}\right) \cap L^{2}\left(0, T ; H^{1}(\Omega)^{2}\right)$.

Proof. The proof is based on the results of Grisvard [9] and Temam [17].

The fully-discrete scheme for (4.1)-(4.4) is: Find $\left(v_{\eta}^{n+1}, q_{\eta}^{n+1}\right)$ with values in $X_{\eta} \times M_{\eta}$, for each $0 \leq n \leq N-1$, solution of:

$$
\begin{gathered}
\forall z_{\eta} \in X_{\eta}, \frac{1}{k}\left(v_{\eta}^{n+1}-v_{\eta}^{n}, z_{\eta}\right)+\nu\left(\nabla v_{\eta}^{n+1}, \nabla z_{\eta}\right)-\left(q_{\eta}^{n+1}, \operatorname{div} z_{\eta}\right)=\left(g^{n+1}, z_{\eta}\right), \\
\forall q_{\eta} \in M_{\eta},\left(q_{\eta}, \operatorname{div} v_{\eta}^{n+1}\right)=0, \\
v_{\eta}^{0}=0 \text { in } \Omega,
\end{gathered}
$$

where $g^{n+1}$ is the same approximation as in (3.1). 
This linear problem has a unique solution that satisfies the following error estimate:

Lemma 4.2. Let $\Omega$ be a convex domain, $g \in L^{2}(\Omega \times] 0, T[)^{2}, g^{\prime} \in L^{2}\left(0, T ; H^{-1}(\Omega)^{2}\right)$ and $g(0) \in L^{2}(\Omega)^{2}$. Then, there exists a constant $C$ that does not depend on $\eta$ and $k$ such that

$$
\begin{aligned}
& \sup _{0 \leq n \leq N}\left\|v_{\eta}^{n}-v\left(t^{n}\right)\right\|_{L^{2}(\Omega)}+\left(\sum_{n=0}^{N-1}\left\|\left(v_{\eta}^{n+1}-v\left(t^{n+1}\right)\right)-\left(v_{\eta}^{n}-v\left(t^{n}\right)\right)\right\|_{L^{2}(\Omega)}^{2}\right)^{1 / 2} \\
&\left.+\sqrt{\nu}\left(\sum_{n=0}^{N-1} k\left|v_{\eta}^{n+1}-v\left(t^{n+1}\right)\right|_{H^{1}(\Omega)}^{2}\right)\right)^{1 / 2} \leq \\
& C(\eta+k)\left(\|g\|_{L^{2}(\Omega \times] 0, T[)^{2}}+\left\|g^{\prime}\right\|_{L^{2}\left(0, T ; H^{-1}(\Omega)^{2}\right)}+\|g(0)\|_{L^{2}(\Omega)^{2}}\right) .
\end{aligned}
$$

In addition, the solution $\left(v_{\eta}^{n+1}, q_{\eta}^{n+1}\right)$ of $(4.5)-(4.7)$ satisfies:

Lemma 4.3. In addition to the hypotheses of Lemma 4.2, suppose that $q^{\prime} \in L^{2}(\Omega \times] 0, T[)$. There exists a constant $C$ that does not depend on $\eta$ and $k$ such that

$$
\begin{aligned}
\left(\sum_{n=0}^{N-1} k\left\|\frac{\left(v_{\eta}^{n+1}-v\left(t^{n+1}\right)\right)-\left(v_{\eta}^{n}-v\left(t^{n}\right)\right)}{k}\right\|_{L^{2}(\Omega)}^{2}\right)^{1 / 2}+\sqrt{\nu} \sup _{0 \leq n \leq N}\left|v_{\eta}^{n}-v\left(t^{n}\right)\right|_{H^{1}(\Omega)} & \\
& +\sqrt{\nu}\left(\sum_{n=0}^{N-1}\left|\left(v_{\eta}^{n+1}-v\left(t^{n+1}\right)\right)-\left(v_{\eta}^{n}-v\left(t^{n}\right)\right)\right|_{H^{1}(\Omega)}^{2}\right)^{1 / 2} \leq C(\eta+\sqrt{k}) .
\end{aligned}
$$

Remark 4.4. The additional assumption $q^{\prime} \in L^{2}(\Omega \times] 0, T[)$ is due to the fact that the operator $S_{\eta}$ is applied to $v^{\prime}$. Therefore, a.e. on $] 0, T\left[\right.$, we have $\left\|S_{\eta} v^{\prime}-v^{\prime}\right\|_{L^{2}(\Omega)} \leq C \eta\left(\left|v^{\prime}\right|_{H^{1}(\Omega)}+\left\|q^{\prime}\right\|_{L^{2}(\Omega)}\right)$.

This regularity hypothesis on $q^{\prime}$ can be obtained, for example, in the following circumstances:

If $g \in H^{1}\left(0, T ; L^{2}(\Omega)^{2}\right)$ and $g(0) \in V$, then $q^{\prime} \in L^{2}\left(0, T ; H^{1 / 2}(\Omega) / \mathbb{R}\right)$. If in addition, $\Omega$ is convex, then $q^{\prime} \in L^{2}\left(0, T ; H^{1}(\Omega) / \mathbb{R}\right)$.

The proof is based on a method of Galerkin. We can refer to, for example, [6].

The parabolic duality argument $(c f .[18])$ consists in defining the solution $\left(w^{n}, \lambda^{n}\right)$ of the backward semidiscrete Stokes system:

$$
\begin{gathered}
\frac{1}{k}\left(w^{n+1}-w^{n}\right)+\nu \Delta w^{n}-\nabla \lambda^{n}=v_{\eta}^{n}-v\left(t^{n}\right) \text { in } \Omega \\
\operatorname{div} w^{n}=0 \text { in } \Omega \\
w^{n}=0 \text { on } \partial \Omega \\
w^{N+1}=0 \text { in } \Omega
\end{gathered}
$$

where $0 \leq n \leq N$.

For each $n$, knowing $w^{n+1}$, the Stokes problem (4.9)-(4.12) has a unique solution $w^{n} \in H_{0}^{1}(\Omega)^{2}$, $\lambda^{n} \in L_{0}^{2}(\Omega),(c f .[8,17])$.

The next lemma establishes basic estimates for the velocity $w^{n}$ of the backward semi-discrete Stokes problem (4.9)-(4.12). 
Lemma 4.5. Standard arguments give the uniform bounds:

$$
\begin{array}{r}
\sup _{0 \leq n \leq N}\left\|w^{n}\right\|_{L^{2}(\Omega)}+\left(\sum_{n=0}^{N}\left\|w^{n+1}-w^{n}\right\|_{L^{2}(\Omega)}^{2}\right)^{1 / 2}+\sqrt{\nu}\left(\sum_{n=0}^{N} k\left|w^{n}\right|_{H^{1}(\Omega)}^{2}\right)^{1 / 2} \leq \\
\sqrt{\frac{3}{\nu}} S_{2}\left(\sum_{n=0}^{N} k\left\|v_{\eta}^{n}-v\left(t^{n}\right)\right\|_{L^{2}(\Omega)}^{2}\right)^{1 / 2},
\end{array}
$$

where $S_{2}$ is the constant of Poincaré's inequality, and

$$
\begin{array}{r}
\sup _{0 \leq n \leq N} \sqrt{\nu}\left|w^{n}\right|_{H^{1}(\Omega)}+\sqrt{\nu}\left(\sum_{n=0}^{N}\left|w^{n+1}-w^{n}\right|_{H^{1}(\Omega)}^{2}\right)^{1 / 2}+\left(\sum_{n=0}^{N} k\left\|\frac{w^{n+1}-w^{n}}{k}\right\|_{L^{2}(\Omega)}^{2}\right)^{1 / 2} \leq \\
\sqrt{3}\left(\sum_{n=0}^{N} k\left\|v_{\eta}^{n}-v\left(t^{n}\right)\right\|_{L^{2}(\Omega)}^{2}\right)^{1 / 2}
\end{array}
$$

If $\Omega$ is convex, (4.14) implies the uniform bound

$$
\left(\sum_{n=0}^{N} k\left(\left|w^{n}\right|_{H^{2}(\Omega)}^{2}+\left|\lambda^{n}\right|_{H^{1}(\Omega)}^{2}\right)\right)^{1 / 2} \leq C\left(\sum_{n=0}^{N} k\left\|v_{\eta}^{n}-v\left(t^{n}\right)\right\|_{L^{2}(\Omega)}^{2}\right)^{1 / 2},
$$

with a constant $C$ independent of $k$ and $\eta$.

Proof. For the first inequality, we take the scalar product of (4.9) with $z=w^{n}$ and we use the incompressibility condition. This gives

$$
-\frac{1}{k}\left(w^{n+1}-w^{n}, w^{n}\right)+\nu\left|w^{n}\right|_{H^{1}(\Omega)}^{2}=\left(v\left(t^{n}\right)-v_{\eta}^{n}, w^{n}\right) .
$$

Multiplying the above equation by $k$, summing it over $n$ from $i$ to $N$, and applying the Poincaré's inequality, we obtain for any $\varepsilon>0$

$$
\begin{aligned}
\frac{1}{2}\left(\left\|w^{i}\right\|_{L^{2}(\Omega)}^{2}+\sum_{j=i}^{N}\left\|w^{j}-w^{j+1}\right\|_{L^{2}(\Omega)}^{2}\right)+\nu \sum_{j=i}^{N} k\left|w^{j}\right|_{H^{1}(\Omega)}^{2} \leq \\
\\
\frac{1}{2} S_{2}\left(\varepsilon \sum_{j=i}^{N} k\left|w^{j}\right|_{H^{1}(\Omega)}^{2}+\frac{1}{\varepsilon} \sum_{j=i}^{N} k\left\|v\left(t^{j}\right)-v_{\eta}^{j}\right\|_{L^{2}(\Omega)}^{2}\right)
\end{aligned}
$$

where $S_{2}$ is Poincaré's constant.

Then (4.13) follows after the suitable choice of $\varepsilon=\frac{\nu}{S_{2}}$.

For the second inequality, we take the scalar product of (4.9) with $z=\frac{1}{k}\left(w^{n}-w^{n+1}\right)$, we multiply the equation by $k$ and sum it over $n$. As precedently, we obtain:

$$
\begin{aligned}
& \sum_{j=i}^{N} \Delta t\left\|\frac{w^{j}-w^{j+1}}{\Delta t}\right\|_{L^{2}(\Omega)}^{2}+\frac{\nu}{2}\left(\left\|\nabla w^{i}\right\|^{2}+\sum_{j=i}^{N}\left|w^{j}-w^{j+1}\right|_{H^{1}(\Omega)}^{2}\right) \leq \\
& \frac{1}{2}\left(\varepsilon \sum_{j=i}^{N} \Delta t\left\|v\left(t^{j}\right)-v_{\eta}^{j}\right\|_{L^{2}(\Omega)}^{2}+\frac{1}{\varepsilon} \sum_{j=i}^{N} \Delta t\left\|\frac{w^{j}-w^{j+1}}{\Delta t}\right\|_{L^{2}(\Omega)}^{2}\right)
\end{aligned}
$$

Then, (4.14) follows readily after the suitable choice of $\varepsilon=1$. 
Now, we assume that $\Omega$ is convex. Since (4.9)-(4.12) is a steady Stokes problem with right-hand side $\left(w^{n+1}-w^{n}\right) / k+v\left(t^{n}\right)-v_{\eta}^{n}$, we have $w^{n} \in H^{2}(\Omega)^{2}, \lambda^{n} \in H^{1}(\Omega)(c f$. [9]) and (4.14) implies also the uniform bound (4.15).

From now on, we assume that $\Omega$ is convex. Using these a priori estimates for the backward Stokes problem, we present a duality argument that allows us to establish that the velocity's error satisfies an estimate of order two in $L^{2}(\Omega \times] 0, T[)^{2}$.

Theorem 4.6. We suppose that there exists a constant $\alpha>0$, independent of $\eta$ and $k$, such that $k \geq \alpha \eta^{2}$. If $g \in L^{2}(\Omega \times] 0, T[)^{2}, g^{\prime} \in L^{2}\left(0, T ; H^{-1}(\Omega)^{2}\right)$ and $g(0) \in L^{2}(\Omega)^{2}$, then there exists a constant $C$, independent of $\eta, k, g, g^{\prime}$ and $g(0)$ such that

$$
\left(\sum_{n=0}^{N} k\left\|v_{\eta}^{n}-v\left(t^{n}\right)\right\|_{L^{2}(\Omega)}^{2}\right)^{1 / 2} \leq C\left(\eta^{2}+k\right)
$$

In particular, if (3.6) holds, then

$$
\left(\sum_{n=0}^{N} k\left\|v_{\eta}^{n}-v\left(t^{n}\right)\right\|_{L^{2}(\Omega)}^{2}\right)^{1 / 2} \leq C \eta^{2}
$$

Proof. Let $e^{n}=v_{\eta}^{n}-v\left(t^{n}\right)$. On one hand, taking the scalar product of (4.9) by $e^{n}$, applying

$$
\sum_{n=0}^{N}\left(a^{n+1}-a^{n}\right) b^{n}=a^{N+1} b^{N}-a^{0} b^{0}+\sum_{n=0}^{N-1} a^{n+1}\left(b^{n}-b^{n+1}\right)
$$

summing over $n$ and inserting $P_{\eta} w^{n+1}$, we obtain

$$
\begin{aligned}
\sum_{n=0}^{N} k\left\|e^{n}\right\|_{L^{2}(\Omega)}^{2} & =\sum_{n=0}^{N-1}\left(w^{n+1}-P_{\eta} w^{n+1}, e^{n}-e^{n+1}\right)+\sum_{n=0}^{N-1}\left(P_{\eta} w^{n+1}, e^{n}-e^{n+1}\right) \\
& -\nu \sum_{n=0}^{N} k\left(\nabla\left(w^{n}-w^{n+1}\right), \nabla e^{n}\right)-\nu \sum_{n=0}^{N} k\left(\nabla w^{n+1}, \nabla e^{n}\right)+\sum_{n=0}^{N} k\left(\lambda^{n}-r_{\eta} \lambda^{n}, \operatorname{div} e^{n}\right)
\end{aligned}
$$

because $w^{N+1}=0, e^{0}=0$ and $\left(r_{\eta} \lambda^{n}, \operatorname{div} e^{n}\right)=\left(r_{\eta} \lambda^{n}, \operatorname{div} v_{\eta}^{n}\right)-\left(r_{\eta} \lambda^{n}, \operatorname{div} v\left(t^{n}\right)\right)=0$.

On the other hand, we integrate (4.1) and (4.5) over $\left[t^{n}, t^{n+1}\right]$ and we take the difference between the resulting equations. This gives

$$
\left(e^{n+1}-e^{n}, \varphi_{\eta}\right)=-\nu \int_{t^{n}}^{t^{n+1}}\left(\nabla\left(v_{\eta}^{n+1}-v(s)\right), \nabla \varphi_{\eta}\right) \mathrm{d} s-\int_{t^{n}}^{t^{n+1}}\left(q(s)-r_{\eta} q(s), \operatorname{div} \varphi_{\eta}\right) \mathrm{d} s
$$


This result is substituted into the second term of the right-hand side of (4.19) with $\varphi_{\eta}=P_{\eta} w^{n+1}$. So (4.19) becomes

$$
\begin{aligned}
\sum_{n=0}^{N} k\left\|e^{n}\right\|_{L^{2}(\Omega)}^{2}= & \sum_{n=0}^{N-1}\left(w^{n+1}-P_{\eta} w^{n+1}, e^{n}-e^{n+1}\right)+\sum_{n=0}^{N-1} \nu \int_{t^{n}}^{t^{n+1}}\left(\nabla\left(v_{\eta}^{n+1}-v(s)\right), \nabla P_{\eta} w^{n+1}\right) \mathrm{d} s \\
& +\sum_{n=0}^{N-1} \int_{t^{n}}^{t^{n+1}}\left(q(s)-r_{\eta} q(s), \operatorname{div} P_{\eta} w^{n+1}\right) \mathrm{d} s-\nu \sum_{n=0}^{N} k\left(\nabla\left(w^{n}-w^{n+1}\right), \nabla e^{n}\right) \\
& -\nu \sum_{n=0}^{N} k\left(\nabla\left(w^{n+1}-P_{\eta} w^{n+1}\right), \nabla e^{n}\right)-\nu \sum_{n=0}^{N} k\left(\nabla P_{\eta} w^{n+1}, \nabla e^{n}\right)+\sum_{n=0}^{N} k\left(\lambda^{n}-r_{\eta} \lambda^{n}, \operatorname{div} e^{n}\right) .
\end{aligned}
$$

Inserting $\pm \nabla v\left(t^{n+1}\right)$ in the second term of the right hand side and using the formula

$$
\int_{t^{n}}^{t^{n+1}} \int_{s}^{t^{n+1}} \nabla v^{\prime}(\tau) \mathrm{d} \tau \mathrm{d} s=\int_{t^{n}}^{t^{n+1}}\left(\tau-t^{n}\right) \nabla v^{\prime}(\tau) \mathrm{d} \tau
$$

this term becomes

$$
\sum_{n=0}^{N-1} \nu \int_{t^{n}}^{t^{n+1}}\left(\nabla e^{n+1}, \nabla P_{\eta} w^{n+1}\right) \mathrm{d} s+\sum_{n=0}^{N-1} \nu \int_{t^{n}}^{t^{n+1}}\left(\tau-t^{n}\right)\left(\nabla v^{\prime}(\tau), \nabla P_{\eta} w^{n+1}\right) \mathrm{d} \tau
$$

The sixth term can be written as follows:

$$
\begin{aligned}
\sum_{n=0}^{N} k\left(\nabla P_{\eta} w^{n+1}, \nabla\left(e^{n}-e^{n+1}\right)\right)+\sum_{n=0}^{N} k\left(\nabla P_{\eta} w^{n+1}, \nabla e^{n+1}\right) & = \\
& \sum_{n=0}^{N} k\left(\nabla P_{\eta}\left(w^{n+1}-w^{n}\right), \nabla e^{n}\right)+\sum_{n=0}^{N} k\left(\nabla P_{\eta} w^{n+1}, \nabla e^{n+1}\right) .
\end{aligned}
$$

Replacing them in (4.20) and using (4.18), we obtain

$$
\begin{aligned}
\sum_{n=0}^{N} k\left\|e^{n}\right\|_{L^{2}(\Omega)}^{2}= & \sum_{n=0}^{N-1}\left(w^{n+1}-P_{\eta} w^{n+1}, e^{n}-e^{n+1}\right)+\sum_{n=0}^{N-1} \nu \int_{t^{n}}^{t^{n+1}}\left(\tau-t^{n}\right)\left(\nabla v^{\prime}(\tau), \nabla P_{\eta} w^{n+1}\right) \mathrm{d} \tau \\
& -\nu \sum_{n=0}^{N} k\left(\nabla\left(P_{\eta} w^{n}-w^{n}\right), \nabla e^{n}\right) \\
& +\sum_{n=0}^{N-1} \int_{t^{n}}^{t^{n+1}}\left(q(s)-r_{\eta} q(s), \operatorname{div}\left(P_{\eta} w^{n+1}-w^{n+1}\right)\right) \mathrm{d} s+\sum_{n=0}^{N} k\left(\lambda^{n}-r_{\eta} \lambda^{n}, \operatorname{div} e^{n}\right) .
\end{aligned}
$$


Denote the terms in the right-hand side of $(4.21)$ by $\left(W_{R H}\right)_{j}, j=1, \ldots, 5$. Using the approximation properties of $P_{\eta}$, applying (4.13) and Lemma 4.2, the first and second terms can be bounded as follows:

$$
\begin{aligned}
\left|\left(W_{R H}\right)_{1}\right| & \leq C \frac{\eta^{2}}{\sqrt{k}}\left(\sum_{n=0}^{N-1}\left\|e^{n}-e^{n+1}\right\|_{L^{2}(\Omega)}^{2}\right)^{1 / 2}\left(\sum_{n=0}^{N-1} k\left\|w^{n+1}\right\|_{H^{2}(\Omega)}^{2}\right)^{1 / 2} \\
& \leq \frac{C \eta^{2}}{\sqrt{k}}(\eta+k)\left(\sum_{n=0}^{N-1} k\left\|e^{n}\right\|_{L^{2}(\Omega)}^{2}\right)^{1 / 2} \\
\left|\left(W_{R H}\right)_{2}\right| & \leq \frac{k}{\sqrt{3}}\left\|v^{\prime}\right\|_{L^{2}\left(0, T ; H^{1}(\Omega)^{2}\right)}\left(\sum_{n=0}^{N-1} k\left\|\nabla P_{\eta} w^{n+1}\right\|_{L^{2}(\Omega)}^{2}\right)^{1 / 2} \\
& \leq \frac{C}{\sqrt{3}} k\left\|v^{\prime}\right\|_{L^{2}\left(0, T ; H^{1}(\Omega)^{2}\right)}\left(\sum_{n=0}^{N} k\left\|e^{n}\right\|_{L^{2}(\Omega)}^{2}\right)^{1 / 2} .
\end{aligned}
$$

Owing to Lemma 4.2 and (4.15), the third term can be bounded by:

$$
\left|\left(W_{R H}\right)_{3}\right| \leq C \eta(\eta+k)\left(\sum_{n=0}^{N} k\left\|e^{n}\right\|_{L^{2}(\Omega)}^{2}\right)^{1 / 2}
$$

Finally, the terms related to the pressure are bounded by:

$$
\begin{aligned}
\left|\left(W_{R H}\right)_{4}\right| & \leq C \eta\left(\sum_{n=0}^{N-1} k\left\|q-r_{\eta} q\right\|_{L^{2}(\Omega)}^{2}\right)^{1 / 2}\left(\sum_{n=0}^{N-1} k\left\|w^{n+1}\right\|_{H^{2}(\Omega)}^{2}\right)^{1 / 2} \\
& \leq C \eta^{2}\|q\|_{L^{2}\left(0, T ; H^{1}(\Omega)\right)}\left(\sum_{n=0}^{N-1} k\left\|e^{n}\right\|_{L^{2}(\Omega)}^{2}\right)^{1 / 2} \cdot \\
\left|\left(W_{R H}\right)_{5}\right| & \leq \eta\left(\sum_{n=0}^{N} k\left|\lambda^{n}\right|_{H^{1}(\Omega)}^{2}\right)^{1 / 2}\left(\sum_{n=0}^{N} k\left|e^{n}\right|_{H^{1}(\Omega)}^{2}\right)^{1 / 2} \\
& \leq C \eta(\eta+k)\left(\sum_{n=0}^{N} k\left\|e^{n}\right\|_{L^{2}(\Omega)}^{2}\right)^{1 / 2} \cdot
\end{aligned}
$$

Substituting these inequalities into (4.21) we obtain (4.16).

If (3.6) holds, then (4.16) implies (4.17).

Now, we split $u_{\eta}^{n}-u\left(t^{n}\right)$ into a linear contribution, $v_{\eta}^{n}-u\left(t^{n}\right)$ and a non-linear one $u_{\eta}^{n}-v_{\eta}^{n}$. Here $v_{\eta}^{n+1}$ is the solution of the Stokes problem (4.5)-(4.7) with $g=f-u \cdot \nabla u$. Therefore, $v=u$ and $v_{\eta}^{n+1}$ solves the discrete problem

$$
\frac{1}{k}\left(v_{\eta}^{n+1}-v_{\eta}^{n}, w_{\eta}\right)+\nu\left(\nabla v_{\eta}^{n+1}, \nabla w_{\eta}\right)-\left(q_{\eta}^{n+1}, \operatorname{div} w_{\eta}\right)=\frac{1}{k} \int_{t^{n}}^{t^{n+1}}\left(f(s)-u(s) \cdot \nabla u(s), w_{\eta}\right) \mathrm{d} s
$$

with (4.6)-(4.7) 
On one hand, by assumption (1.8), we have $f-u \cdot \nabla u \in L^{2}(\Omega \times] 0, T[)^{2}$. Therefore, Theorem 4.6 gives

$$
\left(\sum_{n=0}^{N} k\left\|v_{\eta}^{n}-u\left(t^{n}\right)\right\|_{L^{2}(\Omega)}^{2}\right)^{1 / 2} \leq C(f, u, p, \nu, T)\left(\eta^{2}+k\right),
$$

with another constant $C(f, u, p, \nu, T)$ that does not depend on $\eta^{2}$ nor on $k$.

Furthermore, if $p^{\prime}$ belongs to $L^{2}(\Omega \times] 0, T[)$, Lemma 4.3 implies that

$$
\sup _{0 \leq n \leq N}\left|v_{\eta}^{n}-u\left(t^{n}\right)\right|_{H^{1}(\Omega)} \leq C(\eta+\sqrt{k}) .
$$

On the other hand, we prove the following "superconvergence" result for the non-linear part.

Theorem 4.7. Assume that $f \in L^{\infty}\left(0, T ; L^{2}(\Omega)^{2}\right), \quad p^{\prime} \in L^{2}(\Omega \times] 0, T[), \quad u \in \mathcal{C}^{0}\left(0, T ; W^{1,4}(\Omega)^{2}\right)$ and $u^{\prime} \in L^{2}\left(0, T ; H^{1}(\Omega)^{2}\right)$, then there exists a constant $C$ that does not depend on $\eta$ and $k$, such that

$$
\begin{aligned}
\sup _{0 \leq n \leq N}\left\|v_{\eta}^{n}-u_{\eta}^{n}\right\|_{L^{2}(\Omega)}+\left(\sum_{n=0}^{N-1} \|\left(v_{\eta}^{n+1}-u_{\eta}^{n+1}\right)-\right. & \left.\left(v_{\eta}^{n}-u_{\eta}^{n}\right) \|_{L^{2}(\Omega)}^{2}\right)^{1 / 2} \\
& +\sqrt{\nu}\left(\sum_{n=0}^{N-1} k\left|v_{\eta}^{n+1}-u_{\eta}^{n+1}\right|_{H^{1}(\Omega)}^{2}\right)^{1 / 2} \leq C\left(\eta^{2}+k\right) .
\end{aligned}
$$

Proof. By taking the difference between (4.22) and (1.18), we obtain:

$$
\begin{aligned}
\forall \varphi_{\eta} \in V_{\eta}, \frac{1}{k}\left(\left(v_{\eta}^{n+1}-u_{\eta}^{n+1}\right)-\left(v_{\eta}^{n}-u_{\eta}^{n}\right), \varphi_{\eta}\right)+\nu\left(\nabla\left(v_{\eta}^{n+1}-u_{\eta}^{n+1}\right), \nabla \varphi_{\eta}\right)= \\
\frac{1}{k} \int_{t^{n}}^{t^{n+1}}\left[\left(u_{\eta}^{n+1} \cdot \nabla u_{\eta}^{n+1}-u(s) \cdot \nabla u(s), \varphi_{\eta}\right)+\frac{1}{2}\left(\operatorname{div} u_{\eta}^{n+1}, u_{\eta}^{n+1} \cdot \varphi_{\eta}\right)\right] \mathrm{d} s
\end{aligned}
$$

We split $u(s) \cdot \nabla u(s)-u_{\eta}^{n+1} \cdot \nabla u_{\eta}^{n+1}$ as follows:

$$
\begin{aligned}
u(s) \cdot \nabla u(s)-u_{\eta}^{n+1} \cdot \nabla u_{\eta}^{n+1}=\left(u(s)-u\left(t^{n+1}\right)\right) & \cdot \nabla u(s)+u\left(t^{n+1}\right) \cdot \nabla\left(u(s)-u\left(t^{n+1}\right)\right) \\
-\left(u_{\eta}^{n+1}-v_{\eta}^{n+1}\right) \cdot \nabla u_{\eta}^{n+1}-v_{\eta}^{n+1} \cdot \nabla\left(u_{\eta}^{n+1}-v_{\eta}^{n+1}\right)-\left(v_{\eta}^{n+1}-u\left(t^{n+1}\right)\right) \cdot \nabla\left(v_{\eta}^{n+1}-u\left(t^{n+1}\right)\right) & -\left(v_{\eta}^{n+1}-u\left(t^{n+1}\right)\right) \cdot \nabla u\left(t^{n+1}\right)-u\left(t^{n+1}\right) \cdot \nabla\left(v_{\eta}^{n+1}-u\left(t^{n+1}\right)\right),
\end{aligned}
$$

and we split similarly the divergence term. To simplify, we denote by $b(u ; v, w)$ the sum of these two non-linear terms;

$$
b(u ; v, w)=(u \cdot \nabla v, w)+\frac{1}{2}(\operatorname{div} u, v \cdot w) .
$$


Now, we multiply (4.25) by $k$, choose $\varphi_{\eta}=\varphi_{\eta}^{n+1}=v_{\eta}^{n+1}-u_{\eta}^{n+1}$ which belongs to $V_{\eta}$, and sum it over $n=0, \ldots, m-1$. We obtain:

$$
\begin{aligned}
& \frac{1}{2}\left\|\varphi_{\eta}^{m}\right\|_{L^{2}(\Omega)}^{2}+ \frac{1}{2} \sum_{n=0}^{m-1}\left\|\varphi_{\eta}^{n+1}-\varphi_{\eta}^{n}\right\|_{L^{2}(\Omega)}^{2}+\nu \sum_{n=0}^{m-1} k\left|\varphi_{\eta}^{n+1}\right|_{H^{1}(\Omega)}^{2}= \\
& \sum_{n=0}^{m-1} \int_{t^{n}}^{t^{n+1}}\left(\left(u\left(t^{n+1}\right)-u(s)\right) \cdot \nabla u(s), \varphi_{\eta}^{n+1}\right) \mathrm{d} s+\sum_{n=0}^{m-1} \int_{t^{n}}^{t^{n+1}}\left(u\left(t^{n+1}\right) \cdot \nabla\left(u\left(t^{n+1}\right)-u(s)\right), \varphi_{\eta}^{n+1}\right) \mathrm{d} s \\
&- \sum_{n=0}^{m-1} k b\left(\varphi_{\eta}^{n+1} ; u_{\eta}^{n+1}, \varphi_{\eta}^{n+1}\right)+\sum_{n=0}^{m-1} k\left\{b\left(v_{\eta}^{n+1}-u\left(t^{n+1}\right) ; v_{\eta}^{n+1}-u\left(t^{n+1}\right), \varphi_{\eta}^{n+1}\right)\right. \\
&\left.\quad+b\left(v_{\eta}^{n+1}-u\left(t^{n+1}\right) ; u\left(t^{n+1}\right), \varphi_{\eta}^{n+1}\right)\right\}+\sum_{n=0}^{m-1} k b\left(u\left(t^{n+1}\right) ; v_{\eta}^{n+1}-u\left(t^{n+1}\right), \varphi_{\eta}^{n+1}\right)
\end{aligned}
$$

We note $\left(U_{R H}\right)_{i}, i=1, \ldots, 6$, the terms in the right-hand side of (4.26) and set

$$
C_{0}=\|u\|_{L^{\infty}\left(0, T ; L^{4}(\Omega)^{2}\right)} \text { and } \widehat{C}=\left\|u^{\prime}\right\|_{L^{2}\left(0, T ; L^{4}(\Omega)^{2}\right)} .
$$

For the first two terms, since div $u=0$, we can write

$$
\begin{gathered}
\left(\left(u\left(t^{n+1}\right)-u(s)\right) \cdot \nabla u(s), \varphi_{\eta}^{n+1}\right)=-\left(\left(u\left(t^{n+1}\right)-u(s)\right) \cdot \nabla \varphi_{\eta}^{n+1}, u(s)\right), \\
\left(u\left(t^{n+1}\right) \cdot \nabla\left(u\left(t^{n+1}\right)-u(s)\right), \varphi_{\eta}^{n+1}\right)=-\left(u\left(t^{n+1}\right) \cdot \nabla \varphi_{\eta}^{n+1}, u\left(t^{n+1}\right)-u(s)\right) .
\end{gathered}
$$

Therefore, for any $\varepsilon_{0}>0$,

$$
\left|\left(U_{R H}\right)_{1}\right| \leq \frac{\widehat{C}}{2 \sqrt{3}}\left\{\frac{C_{0}^{2}}{\varepsilon_{0}} k^{2}+\varepsilon_{0} \sum_{n=0}^{m-1} k\left|\varphi_{\eta}^{n+1}\right|_{H^{1}(\Omega)}^{2}\right\}
$$

with the same bound for $\left(U_{R H}\right)_{2}$.

For the third term, we set $C_{1}=\sup _{0 \leq n \leq N}\left|u_{\eta}^{n}\right|_{H^{1}(\Omega)}$. The two parts are treated similarly and we obtain, for any $\varepsilon_{1}>0$

$$
\left|\left(U_{R H}\right)_{3}\right| \leq 2^{1 / 2} C_{1}\left\{\varepsilon_{1} \sum_{n=0}^{m-1} k\left|\varphi_{\eta}^{n+1}\right|_{H^{1}(\Omega)}^{2}+\frac{1}{\varepsilon_{1}} \sum_{n=0}^{m-1} k\left\|\varphi_{\eta}^{n+1}\right\|_{L^{2}(\Omega)}^{2}\right\} .
$$

In order to bound the last two terms, we use the well-known formula

$$
b(u ; v, w)=\frac{1}{2}\left[\int_{\Omega}(u \cdot \nabla v) \cdot w-\int_{\Omega}(u \cdot \nabla w) \cdot v\right] .
$$

The fourth term is split into two parts that we treat successively: using Lemmas 4.2 and 4.3 , for any $\varepsilon_{2}>0$, we bound the first part as follows:

$$
\begin{aligned}
\left|\left(U_{R H}\right)_{4,1}\right| & =\left|\frac{1}{2} \sum_{n=0}^{m-1} k\left(\left(v_{\eta}^{n+1}-u\left(t^{n+1}\right)\right) \cdot \nabla\left(v_{\eta}^{n+1}-u\left(t^{n+1}\right)\right), \varphi_{\eta}^{n+1}\right)\right| \\
& \leq \frac{C S_{4}^{2}}{2}(\eta+k)\left(\sum_{n=0}^{m-1} k\left|\varphi_{\eta}^{n+1}\right|_{H^{1}(\Omega)}^{2}\right)^{1 / 2}\left(\sum_{n=0}^{m-1} k\left|v_{\eta}^{n+1}-u\left(t^{n+1}\right)\right|_{H^{1}(\Omega)}^{2}\right)^{1 / 2} \\
& \leq \frac{S_{4}^{2}}{2}\left\{\frac{C^{2}}{\varepsilon_{2}}\left(\eta^{4}+k^{3}+\eta^{2} k\right)+\varepsilon_{2} \sum_{n=0}^{m-1} k\left|\varphi_{\eta}^{n+1}\right|_{H^{1}(\Omega)}^{2}\right\}
\end{aligned}
$$


and the second part is bounded exactly as the first part. For any $\varepsilon_{3}>0$,

$$
\begin{aligned}
\left|\left(U_{R H}\right)_{4,2}\right| & =\left|\frac{1}{2} \sum_{n=0}^{m-1} k\left(v_{\eta}^{n+1}-u\left(t^{n+1}\right) \cdot \nabla \varphi_{\eta}^{n+1}, v_{\eta}^{n+1}-u\left(t^{n+1}\right)\right)\right| \\
& \leq \frac{S_{4}^{2}}{2} \sup _{n}\left|v_{\eta}^{n+1}-u\left(t^{n+1}\right)\right|_{H^{1}(\Omega)}\left(\sum_{n=0}^{m-1} k\left|\varphi_{\eta}^{n+1}\right|_{H^{1}(\Omega)}^{2}\right)^{1 / 2}\left(\sum_{n=0}^{m-1} k\left|v_{\eta}^{n+1}-u\left(t^{n+1}\right)\right|_{H^{1}(\Omega)}^{2}\right)^{1 / 2} \\
& \leq \frac{S_{4}^{2}}{2}\left\{\frac{C^{2}}{\varepsilon_{3}}\left(\eta^{4}+k^{3}+\eta^{2} k\right)+\varepsilon_{3} \sum_{n=0}^{m-1} k\left|\varphi_{\eta}^{n+1}\right|_{H^{1}(\Omega)}^{2}\right\} .
\end{aligned}
$$

The fifth term is bounded as the fourth term. Setting $C_{2}=\|u\|_{L^{\infty}\left(0, T ; W^{1,4}(\Omega)^{2}\right)}$, for any $\varepsilon_{4}>0$, the first part is bounded as follows:

$$
\begin{aligned}
\left|\left(U_{R H}\right)_{5,1}\right| & =\left|\sum_{n=0}^{m-1} k\left(\left(v_{\eta}^{n+1}-u\left(t^{n+1}\right)\right) \cdot \nabla\left(v_{\eta}^{n+1}-u\left(t^{n+1}\right)\right), \varphi_{\eta}^{n+1}\right)\right| \\
& \leq S_{4}^{2} \sum_{n=0}^{m-1} k\left(\sup _{n}\left|v_{\eta}^{n+1}-u\left(t^{n+1}\right)\right|_{H^{1}(\Omega)}\right)\left|v_{\eta}^{n+1}-u\left(t^{n+1}\right)\right|_{H^{1}(\Omega)}\left|\varphi_{\eta}^{n+1}\right|_{H^{1}(\Omega)} \\
& \leq \frac{S_{4} C_{2}}{2}\left\{\frac{1}{\varepsilon_{4}}\left(\eta^{4}+k^{2}\right)+\varepsilon_{4} \sum_{n=0}^{m-1} k\left|\varphi_{\eta}^{n+1}\right|_{H^{1}(\Omega)}^{2}\right\}
\end{aligned}
$$

and for any $\varepsilon_{5}>0$, the second part is bounded as follows:

$$
\begin{aligned}
\left|\left(U_{R H}\right)_{5,2}\right| & =\left|\frac{1}{2} \sum_{n=0}^{m-1}\left(\operatorname{div}\left(v_{\eta}^{n+1}-u\left(t^{n+1}\right)\right),\left(v_{\eta}^{n+1}-u\left(t^{n+1}\right)\right) \cdot \varphi_{\eta}^{n+1}\right)\right| \\
& \leq \frac{C_{2}}{4}\left\{\frac{1}{\varepsilon_{5}}\left(k^{2}+\eta^{4}\right)+\varepsilon_{5} \sum_{n=0}^{m-1} k\left|\varphi_{\eta}^{n+1}\right|_{H^{1}(\Omega)}^{2}\right\} .
\end{aligned}
$$

Finally, the last term is bounded by applying Green's formula: For any $\varepsilon_{6}>0$,

$$
\begin{aligned}
\left|\left(U_{R H}\right)_{6}\right| & =\left|\sum_{n=0}^{m-1} k\left(u\left(t^{n+1}\right) \cdot \nabla \varphi_{\eta}^{n+1}, v_{\eta}^{n+1}-u\left(t^{n+1}\right)\right)\right| \\
& =\sum_{n=0}^{m-1} k\left\{\left(\left(v_{\eta}^{n+1}-u\left(t^{n+1}\right)\right) \cdot \nabla u\left(t^{n+1}\right), \varphi_{\eta}^{n+1}\right)-\left(\left(v_{\eta}^{n+1}-u\left(t^{n+1}\right)\right) \cdot \nabla \varphi_{\eta}^{n+1}, u\left(t^{n+1}\right)\right)\right\} \\
& \leq \frac{C_{2}}{2}\left\{\frac{1}{\varepsilon_{6}} \sum_{n=0}^{m-1} k\left\|v_{\eta}^{n+1}-u\left(t^{n+1}\right)\right\|_{L^{2}(\Omega)}^{2}+\varepsilon_{6} \sum_{n=0}^{m-1} k\left|\varphi_{\eta}^{n+1}\right|_{H^{1}(\Omega)}^{2}\right\} \\
& \leq \frac{C_{2}}{2}\left\{\frac{1}{\varepsilon_{6}}\left(\eta^{4}+k^{2}\right)+\varepsilon_{6} \sum_{n=0}^{m-1} k\left|\varphi_{\eta}^{n+1}\right|_{H^{1}(\Omega)}^{2}\right\} .
\end{aligned}
$$

Then (4.26) becomes:

$\frac{1}{2}\left\|v_{\eta}^{m}-u_{\eta}^{m}\right\|_{L^{2}(\Omega)}^{2}+\frac{1}{2} \sum_{n=0}^{m-1}\left\|\left(v_{\eta}^{n+1}-u_{\eta}^{n+1}\right)-\left(v_{\eta}^{n}-u_{\eta}^{n}\right)\right\|_{L^{2}(\Omega)}^{2}+\nu \sum_{n=0}^{m-1} k\left|v_{\eta}^{n+1}-u_{\eta}^{n+1}\right|_{H^{1}(\Omega)}^{2} \leq A+B+D$,

where $A \leq C\left(\eta^{4}+k^{2}\right), B=\gamma_{1} \sum_{n=0}^{m-1} k\left\|v_{\eta}^{n+1}-u_{\eta}^{n+1}\right\|_{L^{2}(\Omega)}^{2}, D=\gamma_{2} \sum_{n=0}^{m-1} k\left|v_{\eta}^{n+1}-u_{\eta}^{n+1}\right|_{H^{1}(\Omega)}^{2}$,

$$
\gamma_{1}=\gamma\left(C, \varepsilon_{1}, \varepsilon_{5}\right), \quad \gamma_{2}=\gamma\left(S_{4}, C_{i}, \varepsilon_{j}, i=1,2, j=0, \ldots, 6\right) .
$$


Then, after a suitable choice of $\varepsilon_{i}$ and applying Gronwall's lemma, the equation becomes:

$$
\left\|v_{\eta}^{m}-u_{\eta}^{m}\right\|_{L^{2}(\Omega)}^{2}+\sum_{n=0}^{m-1}\left\|\left(v_{\eta}^{n+1}-u_{\eta}^{n+1}\right)-\left(v_{\eta}^{n}-u_{\eta}^{n}\right)\right\|_{L^{2}(\Omega)}^{2}+\nu \sum_{n=0}^{m-1} k\left|v_{\eta}^{n+1}-u_{\eta}^{n+1}\right|_{H^{1}(\Omega)}^{2} \leq e^{C T}\left(\eta^{4}+k^{2}\right) .
$$

Then the result follows from this inequality.

Combining Theorems 4.6 and 4.7, we obtain:

Corollary 4.8. Under the assumptions of Theorem 4.7, there exists a constant $C$, that does not depend on $\eta$ and $k$, such that

$$
\left(\sum_{n=0}^{N} k\left\|u\left(t^{n}\right)-u_{\eta}^{n}\right\|_{L^{2}(\Omega)}^{2}\right)^{1 / 2} \leq C\left(\eta^{2}+k\right)
$$

\section{An estimate for the Pressure}

The results of the preceding section allow one to establish an error estimate for the pressure. We start with a general bound.

Lemma 5.1. Under the assumptions of Corollary 3.3, suppose that $p^{\prime} \in L^{2}(\Omega \times] 0, T[)$. Let $\left(u\left(t^{n+1}\right), p\left(t^{n+1}\right)\right)$ and $\left(u_{\eta}^{n+1}, p_{\eta}^{n+1}\right)$ be the respective solutions of $(1.1)-(1.4)$ and $(1.18)-(1.19)$. We have

$$
\begin{aligned}
\left(\sum_{n=0}^{N-1} k\left\|p_{\eta}^{n+1}-r_{\eta} p\left(t^{n+1}\right)\right\|_{L^{2}(\Omega)}^{2}\right)^{1 / 2} \leq & \frac{1}{\beta^{\star}}\left\{S_{2}\left(\sum_{n=0}^{N-1} k\left\|\frac{\left(u_{\eta}^{n+1}-u\left(t^{n+1}\right)\right)-\left(u_{\eta}^{n}-u\left(t^{n}\right)\right)}{k}\right\|_{L^{2}(\Omega)}^{2}\right)^{1 / 2}\right. \\
& \left.+C_{1}(\eta+k)+C_{2} k\left\|p^{\prime}\right\|_{L^{2}(\Omega \times] 0, T[)}+C_{3} \eta\|p\|_{L^{2}\left(0, T ; H^{1}(\Omega)\right)}\right\}
\end{aligned}
$$

where $\beta^{\star}$ is the constant of the inf-sup condition (1.10) and the coefficients $C_{i}, 1 \leq i \leq 3$, are independent of $\eta$ and $k$.

Proof. Integrate (1.1) over $\left[t^{n}, t^{n+1}\right]$, substract (1.18), multiply the result by a test function $w_{\eta}^{n+1}$, insert $r_{\eta} p(s)$ and $r_{\eta} p\left(t^{n+1}\right)$ and sum the resulting equation over $n$ from 0 to $N-1$. This gives

$$
\begin{array}{r}
\sum_{n=0}^{N-1} \int_{t^{n}}^{t^{n+1}}\left(p_{\eta}^{n+1}-r_{\eta} p\left(t^{n+1}\right), \operatorname{div} w_{\eta}^{n+1}\right) \mathrm{d} s=\sum_{n=0}^{N-1}\left\{\left(\varphi^{n+1}\left(t^{n+1}\right)-\varphi^{n}\left(t^{n}\right), w_{\eta}^{n+1}\right)+\nu \int_{t^{n}}^{t^{n+1}}\left(\nabla \varphi^{n+1}(s), \nabla w_{\eta}^{n+1}\right) \mathrm{d} s\right. \\
+\left(\int_{t^{n}}^{t^{n+1}}\left(\varphi^{n+1}(s) \cdot \nabla u_{\eta}^{n+1}, w_{\eta}^{n+1}\right) \mathrm{d} s+\frac{1}{2} \int_{t^{n}}^{t^{n+1}}\left(\operatorname{div} \varphi^{n+1}(s), u_{\eta}^{n+1} \cdot w_{\eta}^{n+1}\right) \mathrm{d} s\right) \\
\left.+\int_{t^{n}}^{t^{n+1}}\left(u(s) \cdot \nabla \varphi^{n+1}(s), w_{\eta}^{n+1}\right) \mathrm{d} s+\int_{t^{n}}^{t^{n+1}}\left(r_{\eta} p(s)-r_{\eta} p\left(t^{n+1}\right), \operatorname{div} w_{\eta}^{n+1}\right) \mathrm{d} s+\int_{t^{n}}^{t^{n+1}}\left(p(s)-r_{\eta} p(s), \operatorname{div} w_{\eta}^{n+1}\right) \mathrm{d} s\right\}
\end{array}
$$

where $\varphi^{i}(\tau)=u_{\eta}^{i}-u(\tau)$.

Owing to the inf-sup condition (1.10), there exists a function $w_{\eta} \in V_{\eta}^{\perp}$ such that

$$
\left(\operatorname{div} w_{\eta}, p_{\eta}^{n+1}-r_{\eta} p\left(t^{n+1}\right)\right)=\left\|p_{\eta}^{n+1}-r_{\eta} p\left(t^{n+1}\right)\right\|_{L^{2}(\Omega)}^{2},\left|w_{\eta}\right|_{H^{1}(\Omega)} \leq \frac{1}{\beta^{\star}}\left\|p_{\eta}^{n+1}-r_{\eta} p\left(t^{n+1}\right)\right\|_{L^{2}(\Omega)} .
$$


Let $\left(P_{R H}\right)_{i}, i=1, \ldots, 6$, denote the terms of the right-hand side of $(5.2)$.

We deduce by standard arguments and by using the estimate (3.2):

$$
\begin{aligned}
\left|\left(P_{R H}\right)_{1}\right| \leq & S_{2}\left(\sum_{n=0}^{N-1} k\left\|\frac{\left(u_{\eta}^{n+1}-u\left(t^{n+1}\right)\right)-\left(u_{\eta}^{n}-u\left(t^{n}\right)\right)}{k}\right\|_{L^{2}(\Omega)}^{2}\right)^{1 / 2}\left(\sum_{n=0}^{N-1} k\left|w_{\eta}^{n+1}\right|_{H^{1}(\Omega)}^{2}\right)^{1 / 2}, \\
\left|\left(P_{R H}\right)_{2}\right| \leq & \nu\left(\sum_{n=0}^{N-1} \int_{t^{n}}^{t^{n+1}}\left|u_{\eta}^{n+1}-u(s)\right|_{H^{1}(\Omega)}^{2} \mathrm{~d} s\right)^{1 / 2}\left(\sum_{n=0}^{N-1} k\left|w_{\eta}^{n+1}\right|_{H^{1}(\Omega)}^{2}\right)^{1 / 2} \leq C_{1} \eta\left(\sum_{n=0}^{N-1} k\left|w_{\eta}^{n+1}\right|_{H^{1}(\Omega)}^{2}\right)^{1 / 2}, \\
\left|\left(P_{R H}\right)_{3}\right|= & \left|\sum_{n=0}^{N-1} \int_{t^{n}}^{t^{n+1}}\left\{\left(\left(u_{\eta}^{n+1}-u(s)\right) \cdot \nabla u_{\eta}^{n+1}, w_{\eta}^{n+1}\right)+\frac{1}{2}\left(\operatorname{div}\left(u_{\eta}^{n+1}-u(s)\right), u_{\eta}^{n+1} \cdot w_{\eta}^{n+1}\right)\right\} \mathrm{d} s\right| \\
& \leq S_{4}^{2} \sup _{n}\left\|\nabla u_{\eta}^{n+1}\right\|_{L^{2}(\Omega)}\left(\sum_{n=0}^{N-1} \int_{t^{n}}^{t^{n+1}}\left|\varphi^{n+1}(s)\right|_{H^{1}(\Omega)}^{2} \mathrm{~d} s\right)^{1 / 2}\left(\sum_{n=0}^{N-1} k\left|w_{\eta}^{n+1}\right|_{H^{1}(\Omega)}^{2}\right)^{1 / 2} \\
& \leq C_{2}(\eta+k)\left(\sum_{n=0}^{N-1} k\left|w_{\eta}^{n+1}\right|_{H^{1}(\Omega)}^{2}\right)^{1 / 2}
\end{aligned}
$$

As far as $\left(P_{R H}\right)_{4}$ is concerned, since

$$
\left(P_{R H}\right)_{4}=-\int_{t^{n}}^{t^{n+1}}\left(u(s) \cdot \nabla w_{\eta}^{n+1}, \varphi^{n+1}(s)\right) \mathrm{d} s,
$$

we have

$$
\begin{aligned}
\left|\left(P_{R H}\right)_{4}\right| & \leq S_{4}^{2}\|u\|_{L^{\infty}\left(0, T ; L^{4}(\Omega)^{2}\right)}\left(\sum_{n=0}^{N-1} \int_{t^{n}}^{t^{n+1}}\left|\varphi^{n+1}(s)\right|_{H^{1}(\Omega)}^{2}\right)^{1 / 2}\left(\sum_{n=0}^{N-1} k\left|w_{\eta}^{n+1}\right|_{H^{1}(\Omega)}^{2}\right)^{1 / 2} \\
& \leq C_{3}(\eta+k)\left(\sum_{n=0}^{N-1} k\left|w_{\eta}^{n+1}\right|_{H^{1}(\Omega)}^{2}\right)^{1 / 2} \cdot \\
\left|\left(P_{R H}\right)_{5}\right| & \leq \frac{C k}{\sqrt{3}}\left\|p^{\prime}\right\|_{L^{2}(\Omega \times] 0, T[)}\left(\sum_{n=0}^{N-1} k\left|w_{\eta}^{n+1}\right|_{H^{1}(\Omega)}^{2}\right)^{1 / 2},
\end{aligned}
$$

and

$$
\left|\left(P_{R H}\right)_{6}\right| \leq C \eta\|p\|_{L^{2}\left(0, T ; H^{1}(\Omega)\right)}\left(\sum_{n=0}^{N-1} k\left|w_{\eta}^{n+1}\right|_{H^{1}(\Omega)}^{2}\right)^{1 / 2} .
$$

Then (5.1) follows easily by substituting these inequalities into (5.2).

We have to estimate $\frac{\left(u_{\eta}^{n+1}-u\left(t^{n+1}\right)\right)-\left(u_{\eta}^{n}-u\left(t^{n}\right)\right)}{k}$ in $L^{2}(\Omega \times] 0, T[)^{2}$. This estimate is proven assuming the triangulation satisfies a milder regularity property than uniform regularity (1.9): in addition to this property, there exists a constant $\tilde{\tau}$ that does not depend on $\eta$ or $k$ such that

$$
\rho_{\min } \geq \tilde{\tau} \eta^{5}, \text { where } \rho_{\min }=\inf _{\kappa \in \mathcal{T}_{\eta}} \rho_{\kappa}
$$

More precisely, this assumption is used in proving that $u_{\eta}^{n}$ is bounded in $L^{\infty}\left(0, T ; W^{1,5 / 2}(\Omega)^{2}\right)$. 
Lemma 5.2. Under the assumptions of Theorem 4.7 and if $\mathcal{T}_{\eta}$ satisfies (5.3), there exists a constant $C$ that depends neither on $\eta$ nor on $k$, such that

$$
\sup _{n}\left|u_{\eta}^{n}\right|_{W^{1,5 / 2}(\Omega)} \leq C
$$

Proof. Lets us sketch the proof. We write

$$
\left|u_{\eta}^{n}\right|_{W^{1,5 / 2}(\Omega)} \leq\left|u_{\eta}^{n}-v_{\eta}^{n}\right|_{W^{1,5 / 2}(\Omega)}+\left|v_{\eta}^{n}-P_{\eta} u\left(t^{n}\right)\right|_{W^{1,5 / 2}(\Omega)}+\left|P_{\eta} u\left(t^{n}\right)-u\left(t^{n}\right)\right|_{W^{1,5 / 2}(\Omega)}+\left|u\left(t^{n}\right)\right|_{W^{1,5 / 2}(\Omega)} .
$$

To evaluate the first and second terms in the right-hand side of the above inequality, we consider the reference element $\widehat{\kappa}$, where all norms are equivalent, revert to the element $\kappa$, sum over all $\kappa \in \mathcal{T}_{\eta}$, apply Jensen's inequality and the regularity of $\mathcal{T}_{\eta}$, we obtain then an inverse inequality. Then, we obtain

$$
\left|u_{\eta}^{n}\right|_{W^{1,5 / 2}(\Omega)} \leq C_{1}+C_{2}\left|u\left(t^{n}\right)\right|_{W^{1,5 / 2}(\Omega)} .
$$

As we have $\sup \left|u\left(t^{n}\right)\right|_{W^{1,5 / 2}(\Omega)} \leq C$, the result follows easily.

Lemma 5.3. Under the assumptions of Theorem 4.7 and Lemma 5.2, there exists a constant $C=C\left(u, u^{\prime}, p^{\prime}, u_{\eta}, \nu\right)$ that does not depend on $\eta$ or on $k$, such that

$$
\begin{aligned}
\left(\sum_{n=0}^{N-1} k\left\|\frac{\left(u_{\eta}^{n+1}-u\left(t^{n+1}\right)\right)-\left(u_{\eta}^{n}-u\left(t^{n}\right)\right)}{k}\right\|_{L^{2}(\Omega)}^{2}\right)^{1 / 2}+\sqrt{\nu} \sup _{0 \leq n \leq N}\left|u_{\eta}^{n}-u\left(t^{n}\right)\right|_{H^{1}(\Omega)} \\
+\sqrt{\nu}\left(\sum_{n=0}^{N-1}\left|\left(u_{\eta}^{n+1}-u\left(t^{n+1}\right)\right)-\left(u_{\eta}^{n}-u\left(t^{n}\right)\right)\right|_{H^{1}(\Omega)}^{2}\right)^{1 / 2} \leq C(\eta+\sqrt{k}) .
\end{aligned}
$$

Proof. The proof is similar to that of Lemma 5.1. But here we also insert $S_{\eta} u(s)$, where $S_{\eta}$ is defined by $\forall(u, p) \in V \times L_{0}^{2}(\Omega), S_{\eta}(u) \in V_{\eta}$,

$$
\forall v_{\eta} \in V_{\eta}, \quad \nu\left(\nabla\left(S_{\eta}(u)-u\right), \nabla v_{\eta}\right)=-\left(p, \operatorname{div} v_{\eta}\right)
$$

and we take $e_{\eta}^{n}=u_{\eta}^{n}-S_{\eta} u\left(t^{n}\right)$.

The operator $S_{\eta}$ satisfies

$$
\left|S_{\eta} u-u\right|_{H^{1}(\Omega)} \leq 2\left|P_{\eta} u-u\right|_{H^{1}(\Omega)}+\frac{1}{\nu}\left\|r_{\eta} p-p\right\|_{L^{2}(\Omega)},
$$

and

In fact, $\forall v_{\eta} \in V_{\eta}$,

$$
\left\|S_{\eta} u-u\right\|_{L^{2}(\Omega)} \leq C \eta\left(\left|S_{\eta} u-u\right|_{H^{1}(\Omega)}+\left\|r_{\eta} p-p\right\|_{L^{2}(\Omega)}\right) .
$$

$$
\nu\left(\nabla\left(S_{\eta} u-P_{\eta} u\right), \nabla v_{\eta}\right)=-\nu\left(\nabla\left(P_{\eta} u-u\right), \nabla v_{\eta}\right)-\left(p-r_{\eta} p, \operatorname{div} v_{\eta}\right)
$$

Then

$$
\left|S_{\eta} u-P_{\eta} u\right|_{H^{1}(\Omega)}^{2} \leq\left|P_{\eta} u-u\right|_{H^{1}(\Omega)}\left|S_{\eta} u-P_{\eta} u\right|_{H^{1}(\Omega)}+\frac{1}{\nu}\left\|p-r_{\eta} p\right\|_{L^{2}(\Omega)}\left|S_{\eta} u-P_{\eta} u\right|_{H^{1}(\Omega)} .
$$


Then, (5.7) follows readily. The inequality (5.8) is obtained by a duality argument.

We obtain

$$
\begin{aligned}
& \sum_{n=0}^{m-1} k\left\|\frac{e_{\eta}^{n+1}-e_{\eta}^{n}}{k}\right\|_{L^{2}(\Omega)}^{2}+\frac{\nu}{2}\left(\left\|\nabla e_{\eta}^{m}\right\|_{L^{2}(\Omega)}^{2}-\left\|\nabla e_{\eta}^{0}\right\|_{L^{2}(\Omega)}^{2}+\sum_{n=0}^{m-1}\left\|\nabla\left(e_{\eta}^{n+1}-e_{\eta}^{n}\right)\right\|_{L^{2}(\Omega)}^{2}\right) \\
& \quad=\sum_{n=0}^{m-1} \int_{t^{n}}^{t^{n+1}}\left(u^{\prime}(s)-S_{\eta} u^{\prime}(s), \frac{e_{\eta}^{n+1}-e_{\eta}^{n}}{k}\right) \mathrm{d} s-\nu \sum_{n=0}^{m-1} \int_{t^{n}}^{t^{n+1}}\left(\nabla S_{\eta} u^{\prime}(s), \nabla\left(\frac{e_{\eta}^{n+1}-e_{\eta}^{n}}{k}\right)\right)\left(s-t^{n}\right) \mathrm{d} s \\
& -\sum_{n=0}^{m-1} \int_{t^{n}}^{t^{n+1}}\left(\left(u_{\eta}^{n+1} \cdot \nabla u_{\eta}^{n+1}-u(s) \cdot \nabla u(s)+\frac{1}{2}\left(\operatorname{div} u_{\eta}^{n+1} \nabla u_{\eta}^{n+1}-\operatorname{div} u(s) \nabla u(s)\right), \frac{e_{\eta}^{n+1}-e_{\eta}^{n}}{k}\right)\right) \mathrm{d} s .
\end{aligned}
$$

Let us estimate the three terms $\left(V_{R H}\right)_{i}, i=1, \ldots, 3$, in the right-hand side of (5.9).

The first term is as follows: For any $\varepsilon_{0}>0$,

$$
\left|\left(V_{R H}\right)_{1}\right| \leq \frac{C}{2 \varepsilon_{0}}\left(\left\|u^{\prime}\right\|_{L^{2}\left(0, T ; H^{1}(\Omega)^{2}\right)}^{2}+\left\|p^{\prime}\right\|_{L^{2}(\Omega \times] 0, T[)}^{2}\right) \eta^{2}+\frac{\varepsilon_{0}}{2} \sum_{n=0}^{m-1} k\left\|\frac{e_{\eta}^{n+1}-e_{\eta}^{n}}{k}\right\|_{L^{2}(\Omega)}^{2} .
$$

Setting $\widehat{C}=\left\|S_{\eta} u^{\prime}\right\|_{L^{2}\left(0, T ; H^{1}(\Omega)^{2}\right)}$, the second term is bounded as follows: For any $\varepsilon_{1}>0$,

$$
\begin{aligned}
\left|\left(V_{R H}\right)_{2}\right| & \leq \frac{\nu k}{\sqrt{3}} \sum_{n=0}^{m-1} k^{1 / 2}\left|\frac{e_{\eta}^{n+1}-e_{\eta}^{n}}{k}\right|_{H^{1}(\Omega)}\left(\int_{t^{n}}^{t^{n+1}}\left|S_{\eta} u^{\prime}(s)\right|_{H^{1}(\Omega)}^{2} \mathrm{~d} s\right)^{1 / 2} \\
& \leq \frac{\nu \widehat{C}^{2}}{2 \sqrt{3} \varepsilon_{1}} k+\frac{\nu \varepsilon_{1}}{2 \sqrt{3}} \sum_{n=0}^{m-1}\left|e_{\eta}^{n+1}-e_{\eta}^{n}\right|_{H^{1}(\Omega)}^{2} .
\end{aligned}
$$

Setting $C_{0}=\|u\|_{L^{\infty}(\Omega \times] 0, T[)^{2}}$, using

$$
\begin{aligned}
& u(s) \cdot \nabla u(s)-u_{\eta}^{n+1} \cdot \nabla u_{\eta}^{n+1}-\frac{1}{2} \operatorname{div} u_{\eta}^{n+1} u_{\eta}^{n+1}= \\
& u(s) \cdot \nabla\left(u(s)-u_{\eta}^{n+1}\right)+\left(u(s)-u_{\eta}^{n+1}\right) \cdot \nabla u_{\eta}^{n+1}+\frac{1}{2} \operatorname{div}\left(u(s)-u_{\eta}^{n+1}\right) u_{\eta}^{n+1},
\end{aligned}
$$

the third term is bounded as follows: For any $\varepsilon_{2}, \varepsilon_{3}>0$,

$$
\left|\left(V_{R H}\right)_{3}\right| \leq\left(\frac{C_{0} C^{\prime}}{2 \varepsilon_{2}}+\frac{C^{\prime \prime} C^{\prime}}{\varepsilon_{3}}\right)\left(\eta^{2}+k^{2}\right)+\left(\frac{C_{0} \varepsilon_{2}}{2}+C^{\prime \prime} \varepsilon_{3}\right) \sum_{n=0}^{m-1} k\left\|\frac{e_{\eta}^{n+1}-e_{\eta}^{n}}{k}\right\|_{L^{2}(\Omega)}^{2} .
$$

Then, choosing suitably the parameters $\varepsilon_{i}$, the equation (5.9) becomes

$$
\begin{aligned}
& \sum_{n=0}^{m-1} k\left\|\frac{e_{\eta}^{n+1}-e_{\eta}^{n}}{k}\right\|_{L^{2}(\Omega)}^{2}+\nu\left\|\nabla e_{\eta}^{m}\right\|_{L^{2}(\Omega)}^{2}-\nu\left\|\nabla e_{\eta}^{0}\right\|_{L^{2}(\Omega)}^{2}+\nu \sum_{n=0}^{m-1}\left\|\nabla\left(e_{\eta}^{n+1}-e_{\eta}^{n}\right)\right\|_{L^{2}(\Omega)}^{2} \\
& \leq C\left(\eta^{2}+k\right) .
\end{aligned}
$$

Finally (5.5) follows readily from this result and by applying a triangular inequality and $S_{\eta}$ 's properties. 
From these three lemmas, we easily derive an estimate of order one of the pressure.

Theorem 5.4. Under the assumptions of Theorem 4.7 and Lemma 5.2, there exists a constant $C$ that does not depend on $\eta$ nor on $k$, such that

$$
\left(\sum_{n=0}^{N-1} k\left\|p\left(t^{n+1}\right)-p_{\eta}^{n+1}\right\|_{L^{2}(\Omega)}^{2}\right)^{1 / 2} \leq C(\eta+\sqrt{k}) .
$$

In particular, if (3.6) holds, then

$$
\left(\sum_{n=0}^{N-1} k\left\|p\left(t^{n+1}\right)-p_{\eta}^{n+1}\right\|_{L^{2}(\Omega)}^{2}\right)^{1 / 2} \leq C \eta .
$$

\section{ERror estimate for the SOlution of Step two}

We assume at this stage that we know the solution $u_{H}^{n+1}$ of the first step. Then at each time step, the second step (1.20)-(1.21) is a square system of linear equations in finite dimension, and if $k$ is small enough, it has a unique solution.

This solution satisfies the following error estimate.

Theorem 6.1. Suppose that

$$
\begin{gathered}
u \in L^{\infty}\left(0, T ; H^{1}(\Omega)^{2}\right) \cap L^{2}\left(0, T ; H^{2}(\Omega)^{2}\right) \cap \mathcal{C}^{0}\left(0, T ; W^{1,4}(\Omega)^{2}\right), \\
u^{\prime} \in L^{2}\left(0, T ; H^{1}(\Omega)^{2}\right), p \in L^{\infty}\left(0, T ; H^{1}(\Omega)\right), p^{\prime} \in L^{2}(\Omega \times] 0, T[), f \in L^{2}(\Omega \times] 0, T[)^{2},
\end{gathered}
$$

$f^{\prime} \in L^{2}\left(0, T ; H^{-1}(\Omega)^{2}\right), f(0) \in L^{2}(\Omega)^{2}, \Omega$ convex and that $u_{H}^{n}$ satisfies (3.5). The solution $\left(u_{h}^{n+1}, p_{h}^{n+1}\right)$ of the second step satisfies the following error estimate:

$$
\begin{aligned}
\sup _{0 \leq n \leq N}\left\|u_{h}^{n}-u\left(t^{n}\right)\right\|_{L^{2}(\Omega)}+\left(\sum_{n=0}^{N-1}\left\|\left(u_{h}^{n+1}-u\left(t^{n+1}\right)\right)-\left(u_{h}^{n}-u\left(t^{n}\right)\right)\right\|_{L^{2}(\Omega)}^{2}\right)^{1 / 2} & \\
& +\sqrt{\nu}\left(\sum_{n=0}^{N-1} k\left|u_{h}^{n+1}-u\left(t^{n+1}\right)\right|_{H^{1}(\Omega)}^{2}\right)^{1 / 2} \leq C\left(H^{2}+h+k\right),
\end{aligned}
$$

where $C$ is a constant that does not depend on $h, H$ and $k$.

Proof. On one hand, by choosing $f^{n+1}$ as in (3.1), $u_{h}^{n+1}$ satisfies (1.20). On the other hand, we integrate (1.5) over $\left[t^{n}, t^{n+1}\right]$. Then, taking the difference between the resulting equations, inserting $P_{h} u\left(t^{n+1}\right)$ and $r_{h} p(s)$, choosing $v_{h}=v_{h}^{n+1}=u_{h}^{n+1}-P_{h} u\left(t^{n+1}\right)$, multiplying the equation by $k$ and summing it over $n=0, \ldots, m-1$, we obtain

$$
\begin{aligned}
& \frac{1}{2}(\left.\left\|v_{h}^{m}\right\|_{L^{2}(\Omega)}^{2}+\sum_{n=0}^{m-1}\left\|v_{h}^{n+1}-v_{h}^{n}\right\|_{L^{2}(\Omega)}^{2}\right)+\nu \sum_{n=0}^{m-1} k\left|v_{h}^{n+1}\right|_{H^{1}(\Omega)}^{2} \\
&=\sum_{n=0}^{m-1}\left(\left(u\left(t^{n+1}\right)-P_{h} u\left(t^{n+1}\right)\right)-\left(u\left(t^{n}\right)-P_{h} u\left(t^{n}\right)\right), v_{h}^{n+1}\right)+\nu \sum_{n=0}^{m-1} \int_{t^{n}}^{t^{n+1}}\left(\nabla\left(u(s)-P_{h} u\left(t^{n+1}\right)\right), \nabla v_{h}^{n+1}\right) \mathrm{d} s \\
& \quad+\sum_{n=0}^{m-1} \int_{t^{n}}^{t^{n+1}}\left\{\left(r_{h} p(s)-p(s), \operatorname{div} v_{h}^{n+1}\right)+\left(u(s) \cdot \nabla u(s)-u_{H}^{n+1} \cdot \nabla u_{h}^{n+1}, v_{h}^{n+1}\right)\right\} \mathrm{d} s .
\end{aligned}
$$


Let us estimate the terms $\left(T G_{R H}\right)_{i}, i=1, \ldots, 4$ in the right-hand side of (6.2). The first term is bounded as follows: For any $\varepsilon_{1}>0$,

$$
\left|\left(T G_{R H}\right)_{1}\right| \leq \frac{C h^{2}}{2 \varepsilon_{1}}\left\|u^{\prime}\right\|_{L^{2}\left(0, T ; H^{1}(\Omega)^{2}\right)}^{2}+\frac{\varepsilon_{1}}{2} \sum_{n=0}^{m-1} k\left\|v_{h}^{n+1}\right\|_{L^{2}(\Omega)}^{2} .
$$

The second term is divided into two parts that we treat separately.

The first part is bounded as follows: For any $\varepsilon_{2}>0$,

$$
\begin{aligned}
\left|\left(T G_{R H}\right)_{2,1}\right| & \leq \frac{\nu}{2 \varepsilon_{2}} \sum_{n=0}^{m-1} \int_{t^{n}}^{t^{n+1}}\left|u(s)-P_{h} u(s)\right|_{H^{1}(\Omega)}^{2} \mathrm{~d} s+\frac{\nu \varepsilon_{2}}{2} \sum_{n=0}^{m-1} k\left|v_{h}^{n+1}\right|_{H^{1}(\Omega)}^{2} \\
& \leq \frac{C \nu}{2 \varepsilon_{2}}\|u\|_{L^{2}\left(0, T ; H^{2}(\Omega)^{2}\right)}^{2} h^{2}+\frac{\nu \varepsilon_{2}}{2} \sum_{n=0}^{m-1} k\left|v_{h}^{n+1}\right|_{H^{1}(\Omega)}^{2}
\end{aligned}
$$

and the second part as follows: For any $\varepsilon_{3}>0$,

$$
\left|\left(T G_{R H}\right)_{2,2}\right| \leq \frac{\nu k^{2}}{2 \sqrt{3} \varepsilon_{3}}\left\|u^{\prime}\right\|_{L^{2}\left(0, T ; H^{1}(\Omega)^{2}\right)}^{2}+\frac{\nu \varepsilon_{3}}{2 \sqrt{3}} \sum_{n=0}^{m-1} k\left|v_{h}^{n+1}\right|_{H^{1}(\Omega)}^{2} .
$$

The third term is bounded as follows: For any $\varepsilon_{4}>0$,

$$
\begin{aligned}
\left|\left(T G_{R H}\right)_{3}\right| & \leq \frac{1}{2 \varepsilon_{4}} \sum_{n=0}^{m-1} \int_{t^{n}}^{t^{n+1}}\left\|r_{h} p(s)-p(s)\right\|_{L^{2}(\Omega)}^{2} \mathrm{~d} s+\frac{\varepsilon_{4}}{2} \sum_{n=0}^{m-1} k\left|v_{h}^{n+1}\right|_{H^{1}(\Omega)}^{2} \\
& \leq \frac{C}{2 \varepsilon_{4}}\|p\|_{L^{2}\left(0, T ; H^{1}(\Omega)\right)}^{2} h^{2}+\frac{\varepsilon_{4}}{2} \sum_{n=0}^{m-1} k\left|v_{h}^{n+1}\right|_{H^{1}(\Omega)}^{2} .
\end{aligned}
$$

The non-linear term in the right-hand side can be written as follows:

$$
\begin{aligned}
u(s) \cdot \nabla u(s)-u_{H}^{n+1} \cdot \nabla u_{h}^{n+1}= & \left(u(s)-u_{H}^{n+1}\right) \cdot \nabla u(s)+u_{H}^{n+1} \cdot \nabla\left(u(s)-P_{h} u\left(t^{n+1}\right)\right) \\
& -u\left(t^{n+1}\right) \cdot \nabla v_{h}^{n+1}-\left(u_{H}^{n+1}-u\left(t^{n+1}\right)\right) \cdot \nabla v_{h}^{n+1} .
\end{aligned}
$$

We study the four parts of the non-linear term $(N L)_{i}, i=1, \ldots, 4$, separately. The first part is treated as follows: For any $\varepsilon_{5}>0$,

$$
\begin{aligned}
& \left|\sum_{n=0}^{m-1} \int_{t^{n}}^{t^{n+1}}\left((N L)_{1}, v_{h}^{n+1}\right) \mathrm{d} s\right| \leq \\
& \frac{S_{4}}{2}\|u\|_{L^{\infty}\left(0, T ; W^{1,4}(\Omega)^{2}\right)}\left(\frac{1}{\varepsilon_{5}} \sum_{n=0}^{m-1} \int_{t^{n}}^{t^{n+1}}\left\|u(s)-u_{H}^{n+1}\right\|_{L^{2}(\Omega)}^{2} \mathrm{~d} s+\varepsilon_{5} \sum_{n=0}^{m-1} k\left|v_{h}\right|_{H^{1}(\Omega)}^{2}\right) \\
& \leq \frac{S_{4}}{2}\|u\|_{L^{\infty}\left(0, T ; W^{1,4}(\Omega)^{2}\right)}\left(\frac{C}{\varepsilon_{5}}\left(H^{4}+k^{2}\right)+\varepsilon_{5} \sum_{n=0}^{m-1} k\left|v_{h}\right|_{H^{1}(\Omega)}^{2}\right) .
\end{aligned}
$$

The second term bound is divided into two parts:

$$
\sum_{n=0}^{m-1} \int_{t^{n}}^{t^{n+1}}\left((N L)_{2}, v_{h}^{n+1}\right) \mathrm{d} s=\sum_{n=0}^{m-1} \int_{t^{n}}^{t^{n+1}}\left((N L)_{2,1}+(N L)_{2,2}, v_{h}^{n+1}\right) \mathrm{d} s
$$


with for any $\varepsilon_{6}, \varepsilon_{7}>0$,

$$
\begin{aligned}
\left|\sum_{n=0}^{m-1} \int_{t^{n}}^{t^{n+1}}\left((N L)_{2,1}, v_{h}^{n+1}\right) \mathrm{d} s\right| & =\left|\sum_{n=0}^{m-1} \int_{t^{n}}^{t^{n+1}}\left(u_{H}^{n+1} \cdot \nabla\left(u(s)-P_{h} u(s)\right), v_{h}^{n+1}\right) \mathrm{d} s\right| \\
& \leq \frac{S_{4}^{2}\left(\sup _{n}\left|u_{H}^{n}\right|_{H^{1}(\Omega)}\right)}{2}\left\{\frac{C}{\varepsilon_{6}} h^{2}+\varepsilon_{6} \sum_{n=0}^{m-1} k\left|v_{h}^{n+1}\right|_{H^{1}(\Omega)}^{2}\right\},
\end{aligned}
$$

and

$$
\begin{aligned}
\left|\sum_{n=0}^{m-1} \int_{t^{n}}^{t^{n+1}}\left((N L)_{2,2}, v_{h}^{n+1}\right) \mathrm{d} s\right| & =\left|\sum_{n=0}^{m-1} \int_{t^{n}}^{t^{n+1}}\left(u_{H}^{n+1} \cdot \nabla P_{h}\left(u(s)-u\left(t^{n+1}\right)\right), v_{h}^{n+1}\right) \mathrm{d} s\right| \\
& \leq \frac{S_{4}^{2}\left(\sup _{n}\left|u_{H}^{n}\right|_{H^{1}(\Omega)}\right)}{2 \sqrt{3}}\left\{\frac{\left\|u^{\prime}\right\|_{L^{2}\left(0, T ; H^{1}(\Omega)^{2}\right)}^{2}}{\varepsilon_{7}}+k_{7} \sum_{n=0}^{m-1} k\left|v_{h}^{n+1}\right|_{H^{1}(\Omega)}^{2}\right\} .
\end{aligned}
$$

The third term vanishes:

$$
\left|\sum_{n=0}^{m-1} \int_{t^{n}}^{t^{n+1}}\left((N L)_{3}, v_{h}^{n+1}\right) \mathrm{d} s\right|=0 .
$$

Finally, the last part is bounded as follows: For any $\varepsilon_{8}>0$,

$$
\begin{aligned}
\left|\sum_{n=0}^{m-1} \int_{t^{n}}^{t^{n+1}}\left((N L)_{4}, v_{h}^{n+1}\right) \mathrm{d} s\right| & =\left|\sum_{n=0}^{m-1} \int_{t^{n}}^{t^{n+1}}\left(\left(u_{H}^{n+1}-u\left(t^{n+1}\right)\right) \cdot \nabla v_{h}^{n+1}, v_{h}^{n+1}\right) \mathrm{d} s\right| \\
& \leq \frac{S_{4} C}{2^{3 / 4}}\left\{\varepsilon_{8} \sum_{n=0}^{m-1} k\left|v_{h}^{n+1}\right|_{H^{1}(\Omega)}^{2}+\frac{1}{2 \varepsilon_{8}} \sum_{n=0}^{m-1} k\left(\delta\left|v_{h}^{n+1}\right|_{H^{1}(\Omega)}^{2}+\frac{1}{\delta}\left\|v_{h}^{n+1}\right\|_{L^{2}(\Omega)}^{2}\right)\right\} .
\end{aligned}
$$

Then, collecting these inequalities and choosing suitably the parameters $\varepsilon_{i}$ and $\delta$, and applying Gronwall's lemma, we get

$$
\begin{aligned}
\left\|u_{h}^{m}-P_{h} u\left(t^{m}\right)\right\|_{L^{2}(\Omega)}+\left(\sum_{n=0}^{m-1} \|\left(u_{h}^{n+1}-\right.\right. & \left.\left.P_{h} u\left(t^{n+1}\right)\right)-\left(u_{h}^{n}-P_{h} u\left(t^{n}\right)\right) \|_{L^{2}(\Omega)}^{2}\right)^{1 / 2} \\
& +\sqrt{\nu}\left(\sum_{n=0}^{m-1} k\left|u_{h}^{n+1}-P_{h} u\left(t^{n+1}\right)\right|_{H^{1}(\Omega)}^{2}\right)^{1 / 2} \leq C\left(h+H^{2}+k\right) .
\end{aligned}
$$

Then, (6.1) follows readily from the above result and the $P_{h}$ 's properties.

As a consequence, if $h=H^{2}$ and $h \sim k$, then

$$
\begin{aligned}
\sup _{0 \leq n \leq N}\left\|u_{h}^{n}-u\left(t^{n}\right)\right\|_{L^{2}(\Omega)}+\left(\sum_{n=0}^{N-1}\left\|\left(u_{h}^{n+1}-u\left(t^{n+1}\right)\right)-\left(u_{h}^{n}-u\left(t^{n}\right)\right)\right\|_{L^{2}(\Omega)}^{2}\right)^{1 / 2} & \\
& +\sqrt{\nu}\left(\sum_{n=0}^{N-1} k\left|u_{h}^{n+1}-u\left(t^{n+1}\right)\right|_{H^{1}(\Omega)}^{2}\right)^{1 / 2} \leq C h .
\end{aligned}
$$

Finally, we consider the error of the pressure. As in Section 5, the pressure satisfies the following bound. 
Lemma 6.2. Under the assumptions of Theorems 4.7 and 6.1 , let $\left(u\left(t^{n+1}\right), p\left(t^{n+1}\right)\right)$ and $\left(u_{h}^{n+1}, p_{h}^{n+1}\right)$ be the respective solutions of (1.1)-(1.4) and (1.20)-(1.21). We have

$$
\begin{array}{r}
\left(\sum_{n=0}^{N-1} k\left\|p_{h}^{n+1}-r_{h} p\left(t^{n+1}\right)\right\|_{L^{2}(\Omega)}^{2}\right)^{1 / 2} \leq \frac{1}{\beta^{\star}}\left\{S_{2}\left(\sum_{n=0}^{N-1} k\left\|\frac{\left(u_{h}^{n+1}-u\left(t^{n+1}\right)\right)-\left(u_{h}^{n}-u\left(t^{n}\right)\right)}{k}\right\|_{L^{2}(\Omega)}^{2}\right)^{1 / 2}\right. \\
\left.+C\left(H^{2}+h+k\right)\right\},
\end{array}
$$

where $\beta^{\star}$ is the constant of the inf-sup condition (1.10) and the constant $C$ depends on $u, u^{\prime}, p$ et $p^{\prime}$ but does not depend on $H, h$ and $k$.

Proof. The only difference with the proof of Lemma 5.1 concerns the non-linear term. Here we write

$$
\begin{aligned}
u(s) \cdot \nabla u(s)-u_{H}^{n+1} \cdot \nabla u_{h}^{n+1}= & \left(u(s)-u_{H}^{n+1}\right) \cdot \nabla u(s)+\left(u_{H}^{n+1}-u(s)\right) \cdot \nabla\left(u(s)-u_{h}^{n+1}\right) \\
& +u(s) \cdot \nabla\left(u(s)-u_{h}^{n+1}\right),
\end{aligned}
$$

and

$$
\begin{aligned}
& \left\|\left(u(s) \cdot \nabla u(s)-u_{H}^{n+1} \cdot \nabla u_{h}^{n+1}, w_{h}^{n+1}\right)\right\|_{L^{2}(\Omega)} \leq\left\{\left\|u(s)-u_{H}^{n+1}\right\|_{L^{2}(\Omega)}\|u(s)\|_{W^{1,4}(\Omega)}\right. \\
& \left.\quad+\left(\left\|u_{H}^{n+1}-u(s)\right\|_{L^{4}(\Omega)}+\|u(s)\|_{L^{4}(\Omega)}\right)\left|u(s)-u_{h}^{n+1}\right|_{H^{1}(\Omega)}\right\}\left\|w_{h}^{n+1}\right\|_{L^{4}(\Omega)} .
\end{aligned}
$$

Let us estimate the terms that compose the non-linear term. We have

$$
\begin{aligned}
&\left|\sum_{n=0}^{N-1} \int_{t^{n}}^{t^{n+1}}\left(\left(u(s)-u_{H}^{n+1}\right) \cdot \nabla u(s), w_{h}^{n+1}\right) \mathrm{d} s\right| \\
& S_{4}\left(\sup _{s}\|u(s)\|_{W^{1,4}(\Omega)}\right)\left(\sum_{n=0}^{N-1} \int_{t^{n}}^{t^{n+1}}\left\|u(s)-u_{H}^{n+1}\right\|_{L^{2}(\Omega)}^{2} \mathrm{~d} s\right)^{1 / 2}\left(\sum_{n=0}^{N-1} k\left|w_{h}^{n+1}\right|_{H^{1}(\Omega)}^{2}\right)^{1 / 2} \\
& \leq\left(C_{1} k\left\|u^{\prime}\right\|_{L^{2}(\Omega \times] 0, T[)^{2}}+C\left(H^{2}+k\right)\right)\left(\sum_{n=0}^{N-1} k\left|w_{h}^{n+1}\right|_{H^{1}(\Omega)}^{2}\right)^{1 / 2} .
\end{aligned}
$$

Similarly, the second term is bounded as follows:

$$
\begin{aligned}
& \mid \sum_{n=0}^{N-1} \int_{t^{n}}^{t^{n+1}}\left(\left(u_{H}^{n+1}-u(s)\right) \cdot \nabla\left(u(s)-u_{h}^{n+1}\right)+u(s) \cdot \nabla\left(u(s)-u_{h}^{n+1}\right), w_{h}^{n+1}\right) \mathrm{d} s \mid \\
& \leq S_{4}\left(\sup _{n}\left\|u_{H}^{n+1}-u(s)\right\|_{L^{4}(\Omega)}+\sup _{s}\|u(s)\|_{L^{4}(\Omega)}\right)\left(\sum_{n=0}^{N-1} \int_{t^{n}}^{t^{n+1}}\left|u(s)-u_{h}^{n+1}\right|_{H^{1}(\Omega)}^{2} \mathrm{~d} s\right)^{1 / 2} \\
& \times\left(\sum_{n=0}^{N-1} k\left|w_{h}^{n+1}\right|_{H^{1}(\Omega)}^{2}\right)^{1 / 2} \leq C_{2}\left(H^{2}+k+h\right)\left(\sum_{n=0}^{N-1} k\left|w_{h}^{n+1}\right|_{H^{1}(\Omega)}^{2}\right)^{1 / 2} .
\end{aligned}
$$

Then, (6.5) follows readily from these bounds and from the inf-sup condition (1.10). 
Therefore, here again, we must derive an estimate for

$$
\left(\sum_{n=0}^{N-1} k\left\|\frac{\left(u_{h}^{n+1}-u\left(t^{n+1}\right)\right)-\left(u_{h}^{n}-u\left(t^{n}\right)\right)}{k}\right\|_{L^{2}(\Omega)}^{2}\right)^{1 / 2}
$$

Lemma 6.3. Under the assumptions of Theorems 4.7 and 6.1 , and if $\nabla u \in L^{\infty}(\Omega \times] 0, T[)^{2}, \Delta u^{\prime} \in L^{2}(\Omega \times] 0, T[)^{2}$ and $\nabla p^{\prime} \in L^{2}(\Omega \times] 0, T[)$, there exists a constant $C$ that does not depend on $H, h$ and $k$, such that

$$
\begin{aligned}
& \left(\sum_{n=0}^{N-1} k\left\|\frac{\left(u_{h}^{n+1}-u\left(t^{n+1}\right)\right)-\left(u_{h}^{n}-u\left(t^{n}\right)\right)}{k}\right\|_{L^{2}(\Omega)}^{2}\right)^{1 / 2}+\sqrt{\nu} \sup _{0 \leq n \leq N}\left|u_{h}^{n}-u\left(t^{n}\right)\right|_{H^{1}(\Omega)} \\
& \\
& \quad+\sqrt{\nu}\left(\sum_{n=0}^{N-1}\left|\left(u_{h}^{n+1}-u\left(t^{n+1}\right)\right)-\left(u_{h}^{n}-u\left(t^{n}\right)\right)\right|_{H^{1}(\Omega)}^{2}\right)^{1 / 2} \leq C\left(h+H^{2}+k\right) .
\end{aligned}
$$

Proof. As in the proof of Lemma 6.2 , we insert $S_{h} u\left(t^{n+1}\right)$, we set $e_{h}^{n}=u_{h}^{n}-S_{h} u\left(t^{n}\right)$ and we take

$$
v_{h}^{n+1}=\frac{1}{k}\left(e_{h}^{n+1}-e_{h}^{n}\right)=\frac{1}{k}\left(\left(u_{h}^{n+1}-S_{h} u\left(t^{n+1}\right)\right)-\left(u_{h}^{n}-S_{h} u\left(t^{n}\right)\right)\right) .
$$

Thus we obtain

$$
\begin{aligned}
& \frac{1}{k^{2}} \| e_{h}^{n+1}-e_{h}^{n} \|_{L^{2}(\Omega)}^{2}+\frac{\nu}{k}\left(\nabla e_{h}^{n+1}, \nabla\left(e_{h}^{n+1}-e_{h}^{n}\right)\right)= \\
& \frac{1}{k^{2}}\left(\left(S_{h} u\left(t^{n+1}\right)-u\left(t^{n+1}\right)\right)-\left(S_{h} u\left(t^{n}\right)-u\left(t^{n}\right)\right), e_{h}^{n+1}-e_{h}^{n}\right) \\
& \quad+\int_{t^{n}}^{t^{n+1}} \frac{\nu}{k^{2}}\left(\nabla\left(S_{h} u\left(t^{n+1}\right)-S_{h} u(s)\right), \nabla\left(e_{h}^{n+1}-e_{h}^{n}\right)\right) \mathrm{d} s \\
&+\frac{1}{k^{2}} \int_{t^{n}}^{t^{n+1}}\left(u(s) \cdot \nabla u(s)-u_{H}^{n+1} \cdot \nabla u_{h}^{n+1}, e_{h}^{n+1}-e_{h}^{n}\right) \mathrm{d} s .
\end{aligned}
$$

Then by multiplying (6.7) by $k$ and by summing over $n=0, \ldots, m-1$, we obtain the following left-hand side

$$
\sum_{n=0}^{m-1} k\left\|\frac{e_{h}^{n+1}-e_{h}^{n}}{k}\right\|_{L^{2}(\Omega)}^{2}+\frac{\nu}{2} \sum_{n=0}^{m-1}\left\|\nabla\left(e_{h}^{n+1}-e_{h}^{n}\right)\right\|_{L^{2}(\Omega)}^{2}+\frac{\nu}{2}\left(\left\|\nabla e_{h}^{m}\right\|_{L^{2}(\Omega)}^{2}-\left\|\nabla e_{h}^{0}\right\|_{L^{2}(\Omega)}^{2}\right) .
$$

Let us bound the right-hand side of (6.7). The first term is bounded as follows: For any $\varepsilon_{1}>0$, we have

$$
\begin{aligned}
\left|\sum_{n=0}^{m-1} k\left(\frac{\left(S_{h} u\left(t^{n+1}\right)-u\left(t^{n+1}\right)\right)-\left(S_{h} u\left(t^{n}\right)-u\left(t^{n}\right)\right)}{k}, \frac{e_{h}^{n+1}-e_{h}^{n}}{k}\right)\right| \leq \\
\frac{1}{2}\left(\frac{C h^{2}}{\varepsilon_{1}}\left(\left\|u^{\prime}\right\|_{L^{2}\left(0, T ; H^{1}(\Omega)^{2}\right)}^{2}+\left\|p^{\prime}\right\|_{\left.\left.L^{2}(\Omega \times] 0, T\right]\right)}^{2}\right)+\varepsilon_{1} \sum_{n=0}^{N-1} k\left\|\frac{e_{h}^{n+1}-e_{h}^{n}}{k}\right\|_{L^{2}(\Omega)}^{2}\right) .
\end{aligned}
$$


The second term is treated as follows: For any $\varepsilon_{2}>0$, we have

$$
\begin{aligned}
& \left|\nu \sum_{n=0}^{m-1} \int_{t^{n}}^{t^{n+1}}\left(\nabla\left(\int_{s}^{t^{n+1}} \frac{\mathrm{d}}{\mathrm{d} \tau}\left(S_{h}(u)\right) \mathrm{d} \tau\right), \nabla\left(\frac{e_{h}^{n+1}-e_{h}^{n}}{k}\right)\right) \mathrm{d} s\right| \leq \\
& \nu\left|\sum_{n=0}^{m-1}-\int_{t^{n}}^{t^{n+1}}\left(\Delta u^{\prime}(s), \frac{e_{h}^{n+1}-e_{h}^{n}}{k}\right)\left(s-t^{n}\right) \mathrm{d} s+\sum_{n=0}^{m-1} \int_{t^{n}}^{t^{n+1}}\left(\nabla p^{\prime}(s), \frac{e_{h}^{n+1}-e_{h}^{n}}{k}\right)\left(s-t^{n}\right) \mathrm{d} s\right| \\
& \leq \frac{C \nu k^{2}}{2 \sqrt{3} \varepsilon_{2}}\left(\left\|\Delta u^{\prime}\right\|_{L^{2}(\Omega \times] 0, T[)^{2}}+\left\|\nabla p^{\prime}\right\|_{\left.\left.L^{2}(\Omega \times] 0, T\right]\right)}\right)+\frac{\varepsilon_{2} \nu}{2 \sqrt{3}} \sum_{n=0}^{m-1} k\left\|\frac{e_{h}^{n+1}-e_{h}^{n}}{k}\right\|_{L^{2}(\Omega)}^{2} .
\end{aligned}
$$

The third term is divided into four parts. For the first one, we set $\left\|\nabla u\left(t^{n+1}\right)\right\|_{L^{\infty}(\Omega)} \leq C$, and for any $\varepsilon_{3}>0$, we have

$$
\left|\sum_{n=0}^{m-1} k\left(\left(u\left(t^{n+1}\right)-u_{H}^{n+1}\right) \cdot \nabla u\left(t^{n+1}\right), \frac{e_{h}^{n+1}-e_{h}^{n}}{k}\right)\right| \leq \frac{C}{2 \varepsilon_{3}}\left(H^{4}+k^{2}\right)+\frac{C \varepsilon_{3}}{2} \sum_{n=0}^{m-1} k \|\left.\frac{e_{h}^{n+1}-e_{h}^{n}}{k}\right|_{L^{2}(\Omega)} ^{2}
$$

For the second part of the third term, we use the fact that $\|u(t)\|_{L^{\infty}(\Omega)} \leq C$ and $\left|u_{H^{n}}^{n}\right|_{W^{1,5 / 2}(\Omega)} \leq C$ which means that $\left\|u_{H}^{n}\right\|_{L^{\infty}(\Omega)} \leq C$, and we apply (6.1). Then, for any $\varepsilon_{4}>0$, we have

$$
\begin{aligned}
& \left|\sum_{n=0}^{m-1} k\left(\left(u_{H}^{n+1}-u\left(t^{n+1}\right)\right) \cdot \nabla\left(u\left(t^{n+1}\right)-u_{h}^{n+1}\right), \frac{e_{h}^{n+1}-e_{h}^{n}}{k}\right)\right| \\
& \leq \frac{1}{2 \varepsilon_{4}} C\left(H^{4}+h^{2}+k^{2}\right)+\frac{\varepsilon_{4}}{2} \sum_{n=0}^{N-1} k \|\left.\frac{e_{h}^{n+1}-e_{h}^{n}}{k}\right|_{L^{2}(\Omega)} ^{2} .
\end{aligned}
$$

The third part is bounded as the third one. For any $\varepsilon_{5}>0$, we have

$$
\begin{aligned}
& \left|\sum_{n=0}^{m-1} k\left(u\left(t^{n+1}\right) \cdot \nabla\left(u\left(t^{n+1}\right)-u_{h}^{n+1}\right), \frac{e_{h}^{n+1}-e_{h}^{n}}{k}\right)\right| \leq \\
& \frac{C\left(\sup _{n}\left\|u\left(t^{n}\right)\right\|_{L^{\infty}(\Omega)}\right)}{2 \varepsilon_{5}}\left(H^{2}+h+k\right)^{2}+\frac{\varepsilon_{5}}{2} \sum_{n=0}^{m-1} k \|\left.\frac{e_{h}^{n+1}-e_{h}^{n}}{k}\right|_{L^{2}(\Omega)} ^{2} .
\end{aligned}
$$

The last part is split into two parts that we treat successively.

$$
\begin{aligned}
\left|\sum_{n=0}^{m-1} \int_{t^{n}}^{t^{n+1}}\left(u(s) \cdot \nabla u(s)-u\left(t^{n+1}\right) \cdot \nabla u\left(t^{n+1}\right), \frac{e_{h}^{n+1}-e_{h}^{n}}{k}\right) \mathrm{d} s\right| & = \\
& \left|\sum_{n=0}^{m-1} \int_{t^{n}}^{t^{n+1}}\left(\left(u(s)-u\left(t^{n+1}\right)\right) \cdot \nabla u(s)+u\left(t^{n+1}\right) \cdot \nabla\left(u(s)-u\left(t^{n+1}\right)\right), \frac{e_{h}^{n+1}-e_{h}^{n}}{k}\right) \mathrm{d} s\right| .
\end{aligned}
$$


For any $\varepsilon_{6}>0$, we have

$$
\begin{aligned}
& \left|\sum_{n=0}^{m-1} \int_{t^{n}}^{t^{n+1}}\left(\left(u(s)-u\left(t^{n+1}\right)\right) \cdot \nabla u(s), \frac{e_{h}^{n+1}-e_{h}^{n}}{k}\right) \mathrm{d} s\right|= \\
& \left|-\sum_{n=0}^{m-1} \int_{t^{n}}^{t^{n+1}}\left(\left(\int_{t^{n}}^{\tau} u^{\prime}(\tau) \cdot \nabla u(s) \mathrm{d} s\right) \mathrm{d} \tau, \frac{e_{h}^{n+1}-e_{h}^{n}}{k}\right)\right| \leq \\
& \frac{\left\|u^{\prime}\right\|_{L^{\infty}\left(0, T ; L^{4}(\Omega)^{2}\right)}}{3 \varepsilon_{6}}\|\nabla u\|_{L^{2}\left(0, T ; L^{4}(\Omega)^{2}\right)}^{2} k^{2}+\varepsilon_{6} \sum_{n=0}^{m-1} k\left\|\frac{e_{h}^{n+1}-e_{h}^{n}}{k}\right\|_{L^{2}(\Omega)}^{2},
\end{aligned}
$$

and for any $\varepsilon_{7}>0$, we have

$$
\begin{aligned}
& \mid \sum_{n=0}^{m-1} \int_{t^{n}}^{t^{n+1}}\left(u\left(t^{n+1}\right) \cdot \nabla(u(s)-\right.\left.\left.u\left(t^{n+1}\right)\right), \frac{e_{h}^{n+1}-e_{h}^{n}}{k}\right) \mathrm{d} s \mid \\
& \leq\|u\|_{\left.L^{\infty}(\Omega \times] 0, T\right]}\left\{\frac{\varepsilon_{7}}{2} \sum_{n=0}^{m-1} k\left\|\frac{e_{h}^{n+1}-e_{h}^{n}}{k}\right\|_{L^{2}(\Omega)}^{2}+\frac{k^{2}}{2 \varepsilon_{7}}\left\|u^{\prime}\right\|_{L^{2}\left(0, T ; H^{1}(\Omega)^{2}\right)}\right\} .
\end{aligned}
$$

Then (6.6) follows readily after a suitable choice of $\varepsilon_{i}$ and by applying the $S_{h}^{\prime} s$ properties.

These two lemmas yield immediately the following theorem.

Theorem 6.4. Under the assumptions

$$
\begin{gathered}
u \in L^{2}\left(0, T ; H^{2}(\Omega)^{2}\right) \cap L^{\infty}\left(0, T ; H^{1}(\Omega)^{2}\right), u^{\prime} \in L^{2}\left(0, T ; H^{2}(\Omega)^{2}\right), p \in L^{\infty}\left(0, T ; H^{1}(\Omega)\right), \\
p^{\prime} \in L^{2}\left(0, T ; H^{1}(\Omega)\right), f \in L^{2}(\Omega \times] 0, T[)^{2}, f^{\prime} \in L^{2}\left(0, T ; H^{-1}(\Omega)^{2}\right), f(0) \in L^{2}(\Omega)^{2}
\end{gathered}
$$

and $\Omega$ convex, we have

$$
\left(\sum_{n=0}^{N-1} k\left\|p\left(t^{n+1}\right)-p_{h}^{n+1}\right\|_{L^{2}(\Omega)}^{2}\right)^{1 / 2} \leq C\left(h+H^{2}+k\right),
$$

with a constant $C$ that does not depend on $h, H$ and $k$.

Remark 6.5. As a consequence, if $h=H^{2}$ and $h \sim k$, then

$$
\left(\sum_{n=0}^{N-1} k\left\|p\left(t^{n+1}\right)-p_{h}^{n+1}\right\|_{L^{2}(\Omega)}^{2}\right)^{1 / 2} \leq C h .
$$

This analysis is confirmed by numerical results which are presented in the next section.

\section{Numerical Results}

In order to confirm these results numerically, we did several experiments by using the FreeFem ++ software, see [10] for the "mini" element. The first results concern an academic problem, then we present a practical one.

In what follows, we have studied the following problems: we have solved, in one hand, by the two-grid algorithm, a linearized stabilized scheme in time on the coarse grid then on the fine grid, we solved the linearized scheme, in space, around the coarse solution by the method of characteristics, and on the other hand, we have solved the linear scheme in time, with the anti-symmetric term, on one fine grid only. 

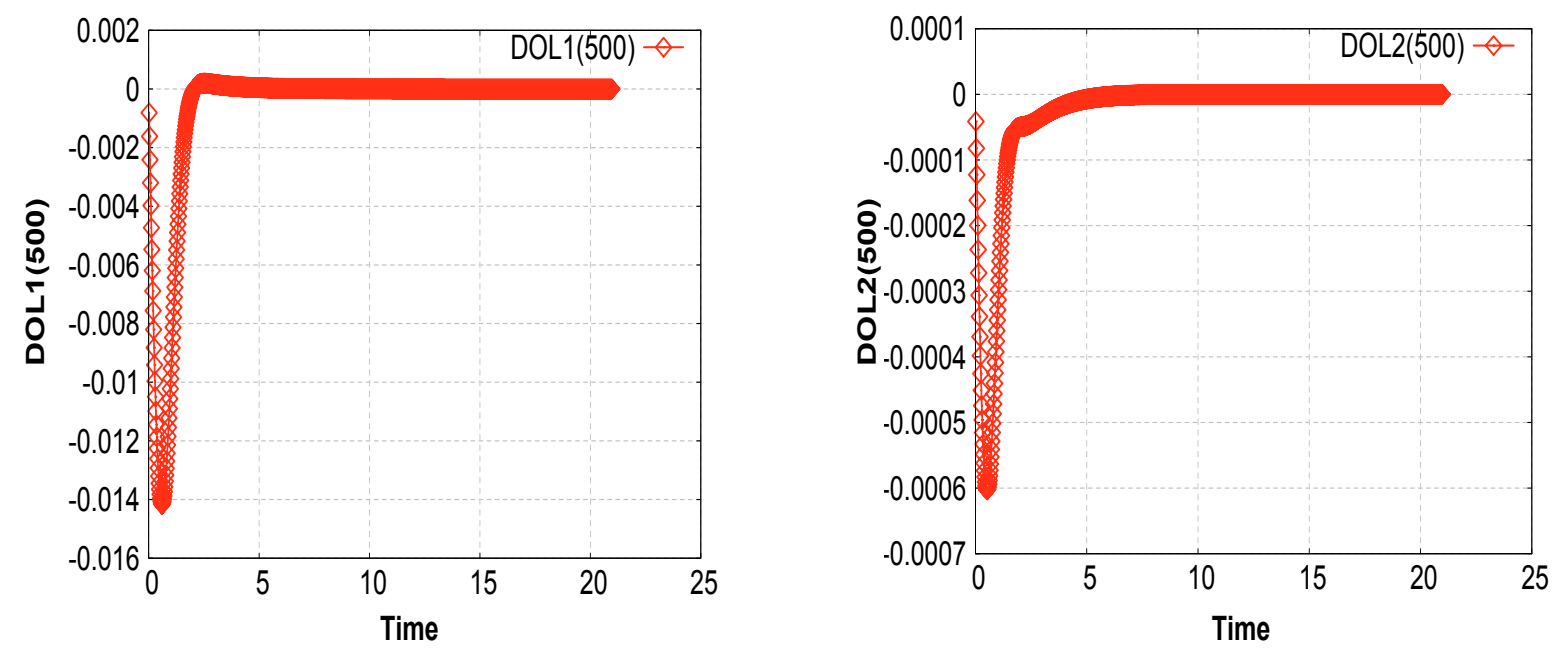

FIGURE 1. The first figure shows a zoom on the evolution of the first component of the degree of liberty 500 in time and the second one is related to the second component.

TABLE 1 . The $L^{2}$ and $H^{1}$ rates.

\begin{tabular}{|c|c|c|c|c|}
\hline meshes & $N^{h}$ & $L^{2}$ rate & $H^{1}$ rate & $L^{2}$ pressure rate \\
\hline \hline$H=1 / 4, h=1 / 16$ & 16 & -2.38503 & -0.587464 & -2.42177 \\
\hline$H=1 / 5, h=1 / 25$ & 25 & -2.62058 & -0.926095 & -2.74363 \\
\hline$H=1 / 6, h=1 / 36$ & 36 & -2.82133 & -1.1698 & -3.03187 \\
\hline$H=1 / 7, h=1 / 49$ & 49 & -2.97968 & -1.33954 & -3.28432 \\
\hline$H=1 / 8, h=1 / 64$ & 64 & -3.10877 & -1.45748 & -3.49683 \\
\hline$H=1 / 9, h=1 / 81$ & 81 & -3.21847 & -1.54327 & -3.6714 \\
\hline
\end{tabular}

On the square domain $] 0,1[\times] 0,1[$, the numerical velocity and the pressure are taken as $(u, p)=($ curl $\psi, p)$, where:

$$
\psi(t, x, y)=t \mathrm{e}^{-t^{2}(x+y)} y^{2}(1-y)^{2} \sin ^{2}(\pi x),
$$

and

$$
p(t, x, y)=t \mathrm{e}^{-t} \cos (2 \pi x) \sin (2 \pi y) .
$$

First of all, we have verified that our problem is stable. In fact, we have fixed the coarse grid $\mathrm{Ng}=7$ points, so the fine one contains $N f=N g^{2}=49$ points and $T=500$ so that the number of iterations becomes nbiter $=T \times N f$.

We present below the evolution of an arbitrary degree of liberty chosen in time (DOL 500). In order to see precisely this evolution, we did a zoom on the velocity's components Figure 1. Next, we have taken $N g=10, N f=N g^{2}, T=1$ and nbiter $=T \times N f$ and we have obtained a color comparison between the exact and numerical solutions of velocity and pressure, Figures 2 and 3.

The graphs related to the velocity's and pressure's error estimations have been studied. The values of these error estimations are given by Table 1 .

So the $L^{2}(\Omega \times] 0, T[)^{2}$ slope is of order 1.1832 and the $L^{2}\left(0, T ; H^{1}(\Omega)^{2}\right)$ slope is of order 1.3569 and the pressure's one in norm $L^{2}(\Omega \times] 0, T[)$ is of order 1.7741, Figures 4 and 5 . 

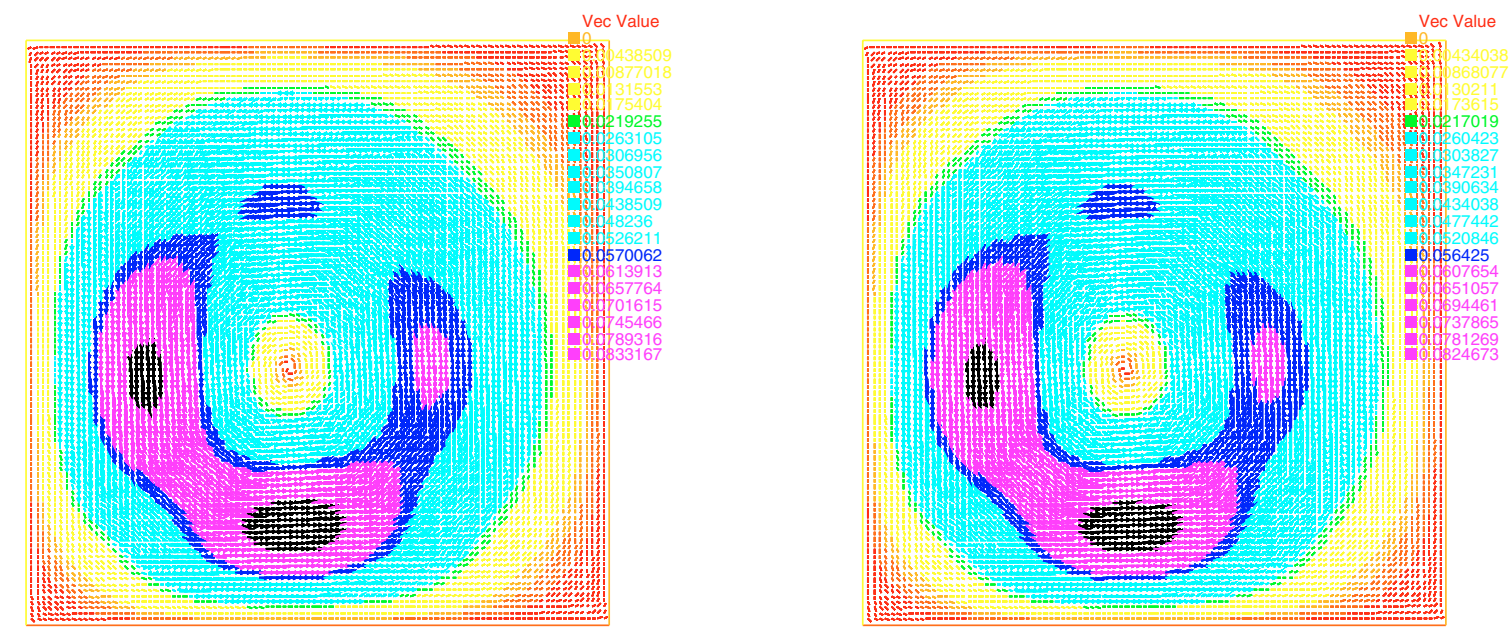

FiguRE 2. The first figure shows the exact velocity's solution and the second is the numerical one.
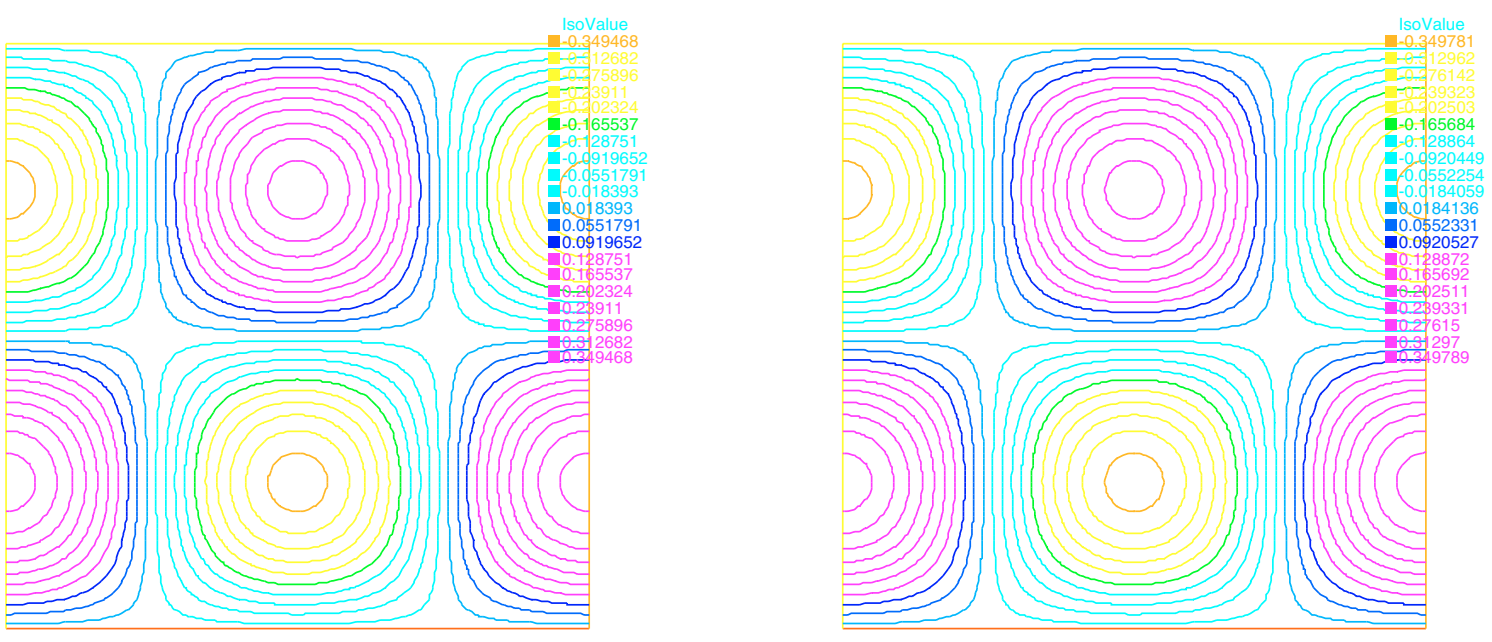

FiguRE 3. The first figure shows the exact pressure's solution and the second is the numerical one.

One of the aims of the resolution of the non-linear Navier-Stokes problem by the two-grid strategy is to gain in computational time. We did a time comparison between the resolution of the linearized scheme in time on one fine grid and the resolution by the two-grid technique. We denote by $t_{2 G}$ and $t_{1 G}$ respectively the time of computation of the resolution by the two-grid method and the resolution on one fine grid. We present these results in Table 2.

Remark 7.1. This gain in time of computation is due to the fact that in the resolution by the two-grid algorithm, we have used the method of characteristics to solve the linearized problem on the fine grid. However, by the resolution on one fine grid only, we have stabilized the problem by adding the anti-symmetric term, in order to avoid the condition on the data $f$. But in this case, we are not able to use the method of characteristics.

If the condition on $f$ is easily assured, in that case, we could have solved the problem on one fine grid only, without the anti-symmetric term, by the method of characteristics. 

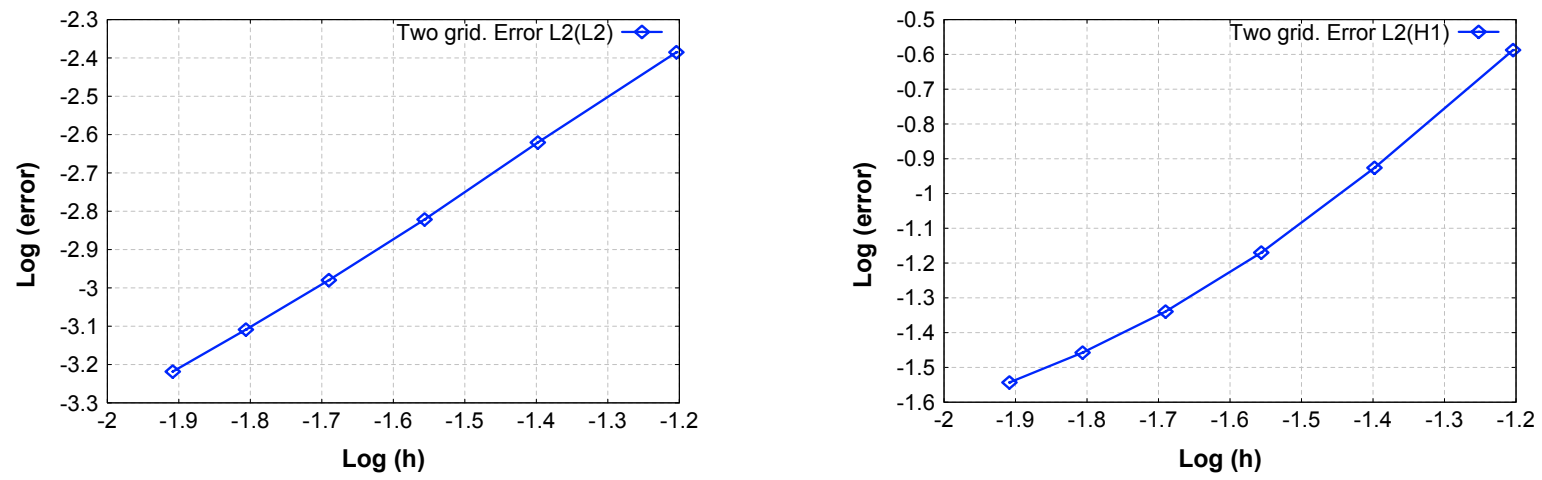

Figure 4 . The first figure shows the error $L^{2}(\Omega \times] 0, T[)^{2}$ and the second one shows the error $L^{2}\left(0, T ; H^{1}(\Omega)^{2}\right)$.

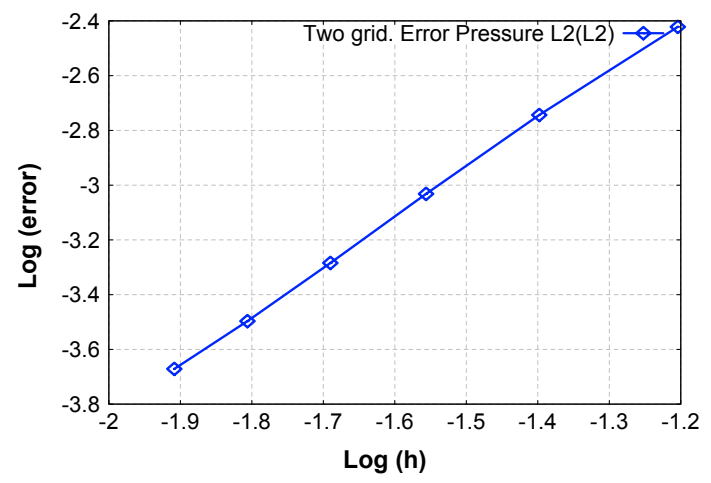

FiguRE 5 . The pressure's error in norm $L^{2}(\Omega \times] 0, T[)$.

TABLE 2. Comparison in computational time CPU.

\begin{tabular}{|c||c||c||c||c||c|}
\hline$N_{g} \times N_{g}$ points & $5 \times 5$ & $6 \times 6$ & $7 \times 7$ & $8 \times 8$ & $9 \times 9$ \\
\hline \hline$t_{1 G}$ (in s) & 85.63 & 236.46 & 597.94 & 1475.75 & 3265.38 \\
\hline \hline$t_{2 G}$ (in s) & 59.05 & 138.844 & 409.047 & 1061.16 & 2448.34 \\
\hline \hline$\frac{t_{2 G}-t_{1 G} \mid}{t_{1 G}}$ (in \%) & 45 & 41.2 & 31.5 & 28.09 & 25.02 \\
\hline
\end{tabular}

In order to validate the two-grid approach, we have treated a more realistic physical problem: we have taken in the square domain $] 0,1[\times] 0,1[$ a cavity. The numerical velocity and the pressure are taken as $(u, p)=(\operatorname{curl} \psi, p)$, where:

$$
\psi(t, x, y)=t \mathrm{e}^{-t^{2}(x+y)} x^{2} y^{2}(x-1)^{2}(y-1)^{2} \sin (4 \pi x)^{2} \sin (4 \pi y)^{2},
$$

and

$$
p(t, x, y)=t \mathrm{e}^{-t} \cos (\pi x) \cos (\pi y) .
$$

Next, we first present a color comparison between the exact and numerical velocity, in both cases: the first numerical velocity is obtained by the resolution of the problem on one fine grid, with the choice of $N f=121$ 

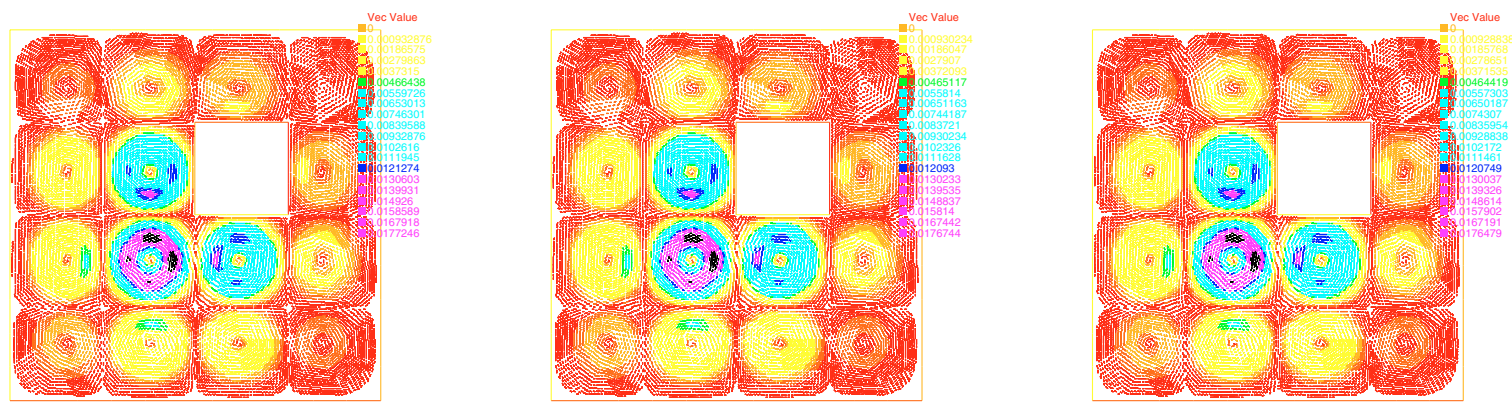

Figure 6. Comparison between the exact and numerical velocity: the first figure shows the exact velocity, the second one the numerical one by the resolution on a fine grid and the last one shows the numerical velocity by the two-grid resolution.
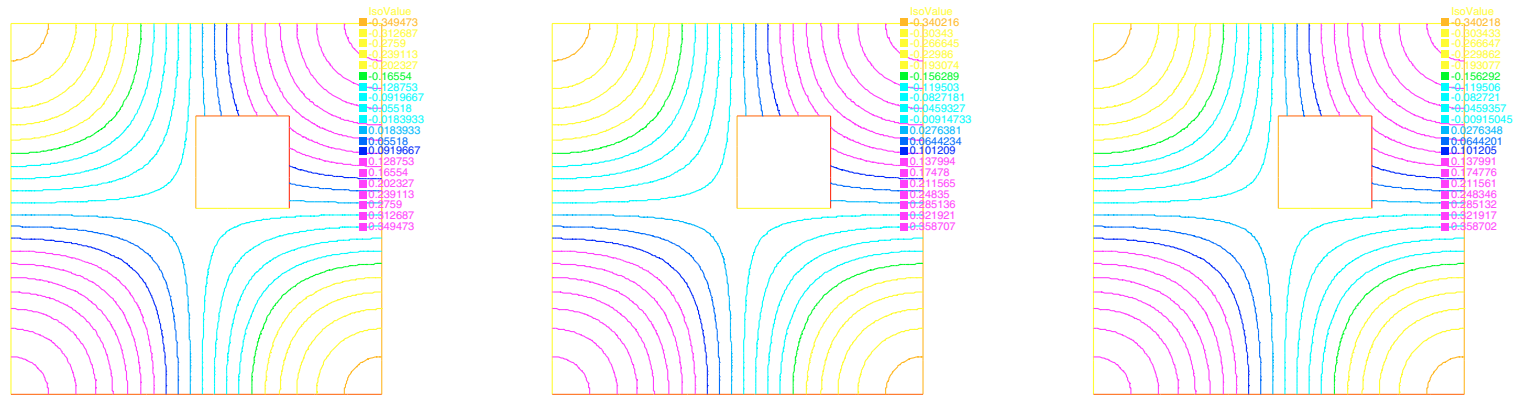

FIGURE 7. Comparison between the exact and numerical pressure: the first figure shows the exact pressure, the second one the numerical one by the resolution on a fine grid and the last one shows the numerical pressure by the two-grid resolution.

and the second one by the two-grid algorithm, with $N g=11, N f=N g^{2}, T=1$ and nbiter $=T \times N f$, cf. Figure 6 .

Secondly, we present a color comparison between the exact and numerical pressure, in both cases: the first numerical pressure is obtained by the resolution of the problem on one fine grid, with the choice of $N f=121$ and the second one by the two-grid algorithm, with $N g=11, N f=N g^{2}, T=1$ and nbiter $=T \times N f$, cf. Figure 7.

The graphs related to the velocity's and pressure's error estimations have been studied and a time comparison between the resolution of the linearized scheme in time on one fine grid and the resolution by the two-grid technique has also been studied. For the resolution on the fine grid only $11 \times 11$, the time of computation is $t_{1 G}=20847 \mathrm{~s}$ and for the two-grid resolution $(N g \times N g=11 \times 11$ and $N f \times N f=121 \times 121), t_{2 G}=18052 \mathrm{~s}$, which gives us a $13,4 \%$ gain in computational time.

Remark 7.2. This gain in computational time is the best that we could achieve in the resolution of the first order time-dependent Navier-Stokes equations by a two-grid scheme. In order to improve our technique, we have studied a second-order scheme in time and space (by using a second-order scheme in time and the second order finite elements of Taylor-Hood) and we have reach a $52 \%$ of gain in computational time, cf. [2]. 
Acknowledgements. The author H. Abboud expresses her deep appreciation and grateful to her Ph.D. advisors Professor V. Girault and Doctor T. Sayah for bringing this problem to her attention, for their support and for their interest in her work.

\section{REFERENCES}

[1] H. Abboud, V. Girault and T. Sayah, Two-grid finite element scheme for the fully discrete time-dependent Navier-Stokes problem. C. R. Acad. Sci. Paris, Ser. I 341 (2005).

[2] H. Abboud, V. Girault and T. Sayah, Second-order two-grid finite element scheme for the fully discrete transient Navier-Stokes equations. Preprint, http://www.ann.jussieu.fr/publications/2007/R07040.html.

[3] R.-A. Adams, Sobolev Spaces. Academic Press, New York (1975).

[4] D. Arnold, F. Brezzi and M. Fortin, A stable finite element for the Stokes equations. Calcolo 21 (1984) 337-344.

[5] P.G. Ciarlet, The Finite Element Method for Elliptic Problems. North-Holland Publishing Company, Amsterdam, New York, Oxford (1978).

[6] V. Girault and J.-L. Lions, Two-grid finite-element schemes for the steady Navier-Stokes problem in polyhedra. Portugal. Math. 58 (2001) 25-57.

[7] V. Girault and J.-L. Lions, Two-grid finite-element schemes for the transient Navier-Stokes equations. ESAIM: M2AN 35 (2001) 945-980.

[8] V. Girault and P.-A. Raviart, Finite Element Methods for the Navier-Stokes Equations. Theory and Algorithms, in Springer Series in Computational Mathematics 5, Springer-Verlag, Berlin (1986).

[9] P. Grisvard, Elliptic Problems in Nonsmooth Domains, Pitman Monographs and Studies in Mathematics 24. Pitman, Boston, (1985).

[10] F. Hecht and O. Pironneau, FreeFem++. See: http://www.freefem.org.

[11] O.A. Ladyzenskaya, The Mathematical Theory of Viscous Incompressible Flow. (In Russian, 1961), First English translation, Gordon \& Breach, New York (1963).

[12] W. Layton, A two-level discretization method for the Navier-Stokes equations. Computers Math. Applic. 26 (1993) 33-38.

[13] W. Layton and W. Lenferink, Two-level Picard-defect corrections for the Navier-Stokes equations at high Reynolds number. Applied Math. Comput. 69 (1995) 263-274.

[14] J.-L. Lions, Quelques Méthodes de Résolution des Problèmes aux Limites Non Linéaires. Dunod, Paris (1969).

[15] J.-L. Lions and E. Magenes, Problèmes aux limites non homogènes et applications I. Dunod, Paris (1968).

[16] J. Nečas, Les méthodes directes en théorie des équations elliptiques. Masson, Paris (1967).

[17] R. Temam, Une méthode d'approximation de la solution des équations de Navier-Stokes. Bull. Soc. Math. France 98 (1968) $115-152$.

[18] M.F. Wheeler, A priori $L_{2}$ error estimates for Galerkin approximations to parabolic partial differential equations. SIAM. J. Numer. Anal. 10 (1973) 723-759.

[19] J. Xu, Some Two-Grid Finite Element Methods. Tech. Report, P.S.U. (1992).

[20] J. Xu, A novel two-grid method of semilinear elliptic equations. SIAM J. Sci. Comput. 15 (1994) 231-237.

[21] J. Xu, Two-grid finite element discretization techniques for linear and nonlinear PDE. SIAM J. Numer. Anal. 33 (1996) 1759-1777. 